\title{
ON THE EXISTENCE OF CONIC KÄHLER-EINSTEIN METRICS
}

\author{
GANG TIAN*, FENG WANG ${ }^{\dagger}$
}

\section{INTRODUCTION}

The Yau-Tian-Donaldson conjecture has been solved for Fano manifolds: If $M$ is a K-polystable Fano manifold, then it admits a Kähler-Einstein metric. Its first proof uses the continuity method through conic Kähler-Einstein metrics with conic angles along a smooth divisor. subsequently, there are proofs by Aubin's continuity method, the Kähler-Ricci flow as well as variational method.

Let us recall conic Kähler metrics along a smooth divisor. First, we have the following model conic metric on $\mathbb{C}^{n}$ :

$$
\omega_{\beta}=\sqrt{-1}\left(\frac{d z_{1} \wedge d \bar{z}_{1}}{\left|z_{1}\right|^{2-2 \beta}}+\sum_{i=2}^{n} d z_{i} \wedge d \bar{z}_{i}\right) .
$$

Definition 1.1. A conic Kähler metric $\omega$ with angle $2 \pi \beta$ along a smooth divisor $D \subset M$ is simply a Kähler metric $\omega$ on $M \backslash D$ such that for each $p \in D$, there is a local coordinate chart $U \subset \mathbb{C}^{n}$ around $p$ with local coordinates $z_{1}, \cdots, z_{n}$ such that $\omega$ is equivalent to the model metric $\omega_{\beta}$ on $U$.

In local coordinates $z_{1}, \cdots, z_{n}$, a conic metric $\omega$ is given by

$$
\omega=\sqrt{-1} \sum_{i, j=1}^{n} g_{i j} d z_{i} \wedge d \bar{z}_{j},
$$

then its Ricci curvature outside $D$ is given by

$$
\operatorname{Ric}(\omega)=-\sqrt{-1} \partial \bar{\partial} \log \operatorname{det}\left(g_{i \bar{j}}\right)
$$

Using this formula, we can deduce that in the sense of currents,

$$
\operatorname{Ric}(\omega)=2 \pi(1-\beta)[D]+\varphi,
$$

where $\varphi$ is a "nice" $(1,1)$-form in certain sense, e.g., it has locally bounded potentials.

Definition 1.2. We say that $\omega$ is a conic Kähler-Einstein metric if

$$
\operatorname{Ric}(\omega)=\mu \omega+2 \pi(1-\beta)[D],
$$

in the sense of currents.

\footnotetext{
* Partially supported by NSFC Grants 11331001 .

$\dagger$ Partially supported by NSFC Grants 11501501 and the Fundamental Research Funds for the Central Universities 2018QNA3001.
} 
If $M$ is a Fano manifold, then there exists a smooth divisor $D \in\left|-\lambda K_{M}\right|$ for some large integer $\lambda>0$. Consider the following equation:

$$
\operatorname{Ric}(\omega)=\mu \omega+2 \pi(1-\beta)[D],
$$

where $\mu+(1-\beta) \lambda=1$. In the course of proving the Yau-Tian-Donaldson conjecture for Fano manifolds, one also showed the solvability of (1) for log-K-stable $(M,(1-\beta) D)$ (cf. [Ti15]). The log K-stability was introduced in [Li11] and extends the notion of K-stability introduced in [Ti97].

A natural question is to consider the existence of conic Kähler-Einstein metric on general log Fano manifolds. A log Fano manifold is a tuple $\left(M, \sum_{i=1}^{k}\left(1-\beta^{i}\right) D_{i}\right)$, where $M$ is a Kähler manifold and $D_{i}$ are normal crossing smooth divisors such that $L$ is an ample $\mathbb{Q}$-line bundle, where $\beta_{i} \in(0,1)$ are rational numbers and

$$
L=K_{M}^{-1}-\sum_{i=1}^{k}\left(1-\beta^{i}\right) D_{i} .
$$

For simplicity, we often write $D$ for $\sum_{i=1}^{k}\left(1-\beta^{i}\right) D_{i}$ and $(M, D)$ for $\left(M, \sum_{i=1}^{k}(1-\right.$ $\left.\left.\beta^{i}\right) D_{i}\right)$.

A metric $\omega$ is called a conic Kähler metric if it is a smooth Kähler metric outside the support $|D|$ of $D$ and for each point $p \in|D|$ where $|D|$ is locally defined by the equation $z_{1} \cdots z_{d}=0$ for some local coordinates $z_{1}, \ldots, z_{n}$, the metric $\omega$ satisfies

$$
C^{-1} \omega_{\text {cone }} \leq \omega \leq C \omega_{\text {cone }},
$$

where $C$ is a positive constant and $\omega_{\text {cone }}$ is the model conic metric with cone angles $2 \pi \beta_{i}$ along $\left\{z_{i}=0\right\}$, that is,

$$
\omega_{\text {cone }}=\sum_{i=1}^{d} \sqrt{-1} \frac{d z_{i} \wedge d \bar{z}_{i}}{\left|z_{i}\right|^{2\left(1-\beta_{i}\right)}}+\sum_{k=d+1}^{n} \sqrt{-1} d z_{i} \wedge d \bar{z}_{i} .
$$

A conic Kähler metric is called a conic Kähler-Einstein metric if it satisfies:

$$
\operatorname{Ric}(\omega)=\omega+2 \pi D,
$$

where $D=\sum_{i=1}^{k}\left(1-\beta^{i}\right) D_{i}$. It is proved in [Ber] that if $(M, D)$ admits a conic Kähler-Einstein metric, then it is log- $K$-polystable as defined in [Li11]. So we want to prove the converse, that is, the $\log K$-polystability is also sufficient for the existence of conic Käler-Einstein metrics with conic angle $2 \pi \beta_{i}$ along each $D_{i}$.

One of our motivations comes from the study of the existence of Kähler-Einstein metrics on $K$-polystable singular Fano variety. Such an existence problem can be reduced to studying the existence and compactness of general conic Käler-Einstein metrics. Let $X$ be a $\mathbb{Q}$-Fano variety. Assume that there exists a log resolution $\mu: M \rightarrow X$ such that

$$
K_{M}=\mu^{*} K_{X}+\sum_{i} a_{i} E_{i}
$$

with $a_{i} \in(-1,0]$. So we have

$$
K_{M}^{-1}=\mu^{*} K_{X}^{-1}+\sum_{i} b_{i} E_{i}
$$


with $b_{i} \in[0,1)$. Then

$$
L_{\epsilon}=\mu^{*} K_{X}^{-1}-\sum_{i} \epsilon_{i} E_{i}=K_{M}^{-1}-\sum_{i}\left(b_{i}+\epsilon_{i}\right) E_{i}
$$

is an ample $\mathbb{Q}$-line bundle for suitable small rational numbers $\epsilon_{i}$. If there is a Kähler-Einstein metric $\omega$ on $X$, then $\mu^{*} \omega$ will be a degenerate conic Kähler-Einstein metric with cone angle $2 \pi\left(1-b_{i}\right)$ along $E_{i}$ in its normal direction:

$$
\operatorname{Ric}\left(\mu^{*} \omega\right)=\mu^{*} \omega+2 \pi \sum_{i} b_{i} E_{i}
$$

So for a $K$-stable $\mathbb{Q}$-Fano variety with such a resolution, we first expect that $\mu^{*} \omega$ can be perturbed to obtain a sequence of conic Kähler Einstein metrics on $M$ :

$$
\operatorname{Ric}\left(\omega_{\epsilon}\right)=\omega_{\epsilon}+2 \pi \sum_{i}\left(b_{i}+\epsilon\right) E_{i},\left[\omega_{\epsilon}\right]=2 \pi c_{1}\left(L_{\epsilon}\right) .
$$

Then we expect that the Gromov-Hausdorff limit of $\left(M, \omega_{\epsilon}\right)$ coincides with the Kähler-Einstein metric on $X$. This is indeed true and we can turn around to use this to construct a Kähler-Einstein metric on $X$.

Our main theorem of this paper is the following

Theorem 1.3. If $\left(M, \Sigma_{i=1}^{k}\left(1-\beta^{i}\right) D_{i}\right)$ is log $K$-polystable, then there exists a conic Kähler-Einstein metric with angle $2 \pi \beta^{i}$ along $D_{i}$.

We will follow the approach in [Ti15] to prove Theorem 1.3. At first, we approximate the conic Kähler-Einstein metric by smooth metrics with Ricci curvature bounded from below. If each $D_{i}$ is semi-ample, then this approximation has been done in [S] by extending the method introduced in [Ti15] which uses the semi-ampleness of $D_{i}$ in a crucial way. However, in our case, the conic divisors $D_{i}$ may not be semi-ample, so we need to use a variational method to do the approximation. With the approximation, we can apply Cheeger-Colding's theory and Cheeger-Colding-Tian's theory. Secondly, adapting the arguments in [Ti15] to our case here, we establish the partial $C^{0}$ estimate. In our new situation here, not all the angles approach $2 \pi$ as one had in the smooth case, this causes some problems of extending certain arguments from the smooth case to our more general cases. At last, we prove the existence of conic Kähler-Einstein metric by using $\log K$ polystability.

\section{SMOOTHING CONIC KäHLER-EINSTEIN METRIC}

In this section, we prove an approximation theorem for general conic Kähler metrics. Our theorem extends the main result in $[\bar{S}]$ under the assumption of semiampleness of the conic divisors.

Let $(M, D)$ be a $\log$ Fano manifold and $\omega$ be a conic Kähler-Einstein metric, where $D=\sum_{i=1}^{k}\left(1-\beta^{i}\right) D_{i}$ and $\beta^{i} \in(0,1)$.

We say that $\omega$ has a $K$-approximation if there is a sequence of smooth metrics $\omega_{i}=\omega+\sqrt{-1} \partial \bar{\partial} \phi_{i}$ satisfying:

i) $\phi_{i}$ converges to 0 uniformly in $M$ and smoothly outside the support $|D|$ of $D$; 
ii) $\operatorname{Ric}\left(\omega_{i}\right) \geq K \omega_{i}$;

iii) $\left(M, \omega_{i}\right)$ converges to $(M, \omega)$ in the Gromov-Hausdorff topology.

Theorem 2.1. Assume that $\operatorname{Aut}^{0}(M, D)=1$ and $\left(1-K_{i}\right) L+\left(1-\beta^{i}\right) D_{i}$ are all semi-positive for some $K_{i} \leq 1(1 \leq i \leq k)$, then $\omega$ has a $K$-approximation, where $K=\Sigma\left(K_{i}-1\right)+1$.

It is easy to see that the condition in Theorem 2.1 is necessary when $k=1$. Now we fix a smooth metric $\omega_{0} \in 2 \pi c_{1}(L)$. For each $i$, since $\left(1-K_{i}\right) L+\left(1-\beta^{i}\right) D_{i}$ is semi-positive, there is a smooth Hermitian metric $h_{i}$ on $\left[D_{i}\right]$ such that

$$
\left(1-K_{i}\right) \omega_{0}+\left(1-\beta^{i}\right) R\left(h_{i}\right) \geq 0,
$$

where $R\left(h_{i}\right)$ is the curvature form of $h_{i}$. Choose a smooth volume form $\Omega$ such that

$$
\operatorname{Ric}(\Omega)=\omega_{0}+\sum_{i=1}^{k}\left(1-\beta^{i}\right) R\left(h_{i}\right), \int_{M} \Omega=\int_{M} \omega_{0}^{n}=V .
$$

Then the conic Kähler-Einstein metric $\omega$ can be written as $\omega_{0}+\sqrt{-1} \partial \bar{\partial} \phi_{K E}$ for some function $\phi_{K E}$ satisfying:

$$
\left(\omega_{0}+\sqrt{-1} \partial \bar{\partial} \phi_{K E}\right)^{n}=e^{-\phi_{K E}}\left(\prod_{i=1}^{k}\left|S_{i}\right|_{h_{i}}^{-2\left(1-\beta^{i}\right)}\right) \Omega,
$$

where $S_{i}$ is a defining section of $D_{i}$. Consider the following perturbed equation:

$$
\left(\omega_{0}+\sqrt{-1} \partial \bar{\partial} \phi_{\delta}\right)^{n}=\lambda e^{-\phi_{\delta}}\left(\prod_{i=1}^{k}\left(\left|S_{i}\right|_{h_{i}}^{2}+\delta e^{-\frac{1-K_{i}}{1-\beta^{i}} \phi_{\delta}}\right)^{-\left(1-\beta^{i}\right)}\right) \Omega .
$$

where $\lambda$ is some positive constant which may depend on $\delta$.

Proposition 2.2. There are constants $a, b, \delta_{0}>0$ depending only on $\left.\left(M, \omega_{0}\right), \Omega, S_{i}, h_{i}\right)$ such that for any $\delta \leq \delta_{0}$, (3) has a solution $\omega_{\delta}$ with some $\lambda \in[a, b]$.

We will use the variational method to prove this proposition. Following [BEGZ], we define

$$
P S H_{\text {full }}=\left\{\phi \in P S H\left(M, \omega_{0}\right) \mid \lim _{j \rightarrow \infty} \int_{\phi \leq-j}\left(\omega_{0}+\sqrt{-1} \partial \bar{\partial} \max \{\phi,-j\}\right)^{n}=0\right\}
$$

and

$$
\mathcal{E}^{1}\left(M, \omega_{0}\right)=\left\{\phi \in P S H_{\text {full }}\left(M, \omega_{0}\right) \mid \phi \in L^{1}\left(\omega_{\phi}\right)\right\} .
$$

The topology on these spaces are all weak topology, i.e. the $L^{1}$-topology.

It follows from Lemma 6.4 in [BBGZ]

Lemma 2.3. The map

$$
\mathcal{E}^{1}\left(M, \omega_{0}\right) \rightarrow L^{1}\left(M, \omega_{0}\right): \phi \rightarrow e^{-\phi}
$$

is continuous. 
Now we define

$$
\mathcal{H}=\left\{\phi \in \mathcal{E}^{1}\left(M, \omega_{0}\right) \mid \int_{M} h\left(e^{-\phi}\right) \Omega=\int_{M} h(1) \Omega\right\}
$$

where

$$
h(x)=\int_{0}^{x} \prod_{i=1}^{k}\left(\left|S_{i}\right|_{h_{i}}^{2}+\delta t^{\frac{1-K_{i}}{1-\beta^{i}}}\right)^{-\left(1-\beta^{i}\right)} d t .
$$

By Lemma $2.3, \mathcal{H}$ is a closed subset of $\mathcal{E}^{1}\left(M, \omega_{0}\right)$.

We have two functionals on $\mathcal{H}$ :

$$
\begin{aligned}
& J(\phi)=\frac{1}{V} \int_{M} \phi \omega_{0}^{n}-\frac{1}{(n+1) V} \sum_{i=0}^{n} \int_{M} \phi \omega_{0}^{i} \wedge \omega_{\phi}^{n-i}, \\
& F_{\delta}(\phi)=J(\phi)-\frac{1}{V} \int_{M} \phi \omega_{0}^{n}-\log \left(\int_{M} h\left(e^{-\phi}\right) \Omega\right) .
\end{aligned}
$$

It is easy to see that

$$
F_{\delta}(\phi)=J(\phi)-\frac{1}{V} \int_{M} \phi \omega_{0}^{n}+F_{\delta}(0), F_{\delta}(0)=-\log \int_{M} h(1) \Omega .
$$

For $\delta \leq 1, F_{\delta}(0)$ is uniformly bounded by a constant depending only on $\left(M, \omega_{0}, \Omega, S_{i}, h_{i}\right)$.

Lemma 2.4. $J(\phi)$ is lower semi-continuous on $\mathcal{H}$.

Proof. By Proposition 2.10 in [BEGZ], we know that $J(\phi)$ is lower semi-continuous on $\mathcal{E}^{1}\left(M, \omega_{0}\right)$. Since $\mathcal{H}$ is a closed subset of $\mathcal{E}^{1}\left(M, \omega_{0}\right)$, the lemma is proved.

Now we prove the proposition.

Proof. From Theorem 2.12 in [DR], we know that log Ding functional

$$
F_{0}(\phi)=J(\phi)-\frac{1}{V} \int_{M} \phi \omega_{0}^{n}-\log \left(\int_{M} \prod_{i=1}^{k}\left|S_{i}\right|_{h_{i}}^{-2\left(1-\beta^{i}\right)} e^{-\phi} \Omega\right)
$$

is coercive, that is, for some positive constants $A$ and $B$, we have

$$
F_{0}(\phi) \geq A J(\phi)-B
$$

Clearly, $F_{\delta} \geq F_{0}$, so $F_{\delta}$ is also coercive and consequently, there is a minimizing sequence $\phi_{j}$ of $F_{\delta}$ satisfying:

$$
\lim _{j \rightarrow \infty} F_{\delta}\left(\phi_{j}\right)=\inf _{\phi \in \mathcal{H}} F_{\delta}(\phi) .
$$

By the above coercive inequality, for $j$ sufficiently large, we have

$$
J\left(\phi_{j}\right) \leq \frac{1}{A}\left(F_{\delta}\left(\phi_{j}\right)+B\right) \leq \frac{1}{A}\left(F_{\delta}(0)+B\right)+1,
$$

Hence,

$$
\left|\int_{M} \phi_{j} \omega_{0}^{n}\right| \leq\left|J\left(\phi_{j}\right)\right|+\left|F_{\delta}\left(\phi_{j}\right)\right|+\left|F_{\delta}(0)\right| \leq C\left(A, B, F_{\delta}(0)\right)
$$

So we have

$$
\left|\sup \phi_{j}\right| \leq C\left(A, B, F_{\delta}(0)\right)
$$


From this and (4), we know that $\phi_{j}$ lies in a weakly compact subset $\mathcal{K} \subset \mathcal{E}^{1}\left(M, \omega_{0}\right)$. Note that $\mathcal{K}$ is independent of $\delta$. Hence, by taking a subsequence if necessary, we may assume that $\phi_{j}$ converge to a limit $\phi_{\delta}$ in $\mathcal{E}^{1}\left(M, \omega_{0}\right)$. From Lemma2.4, we know that $\phi_{\delta}$ is a minimizer of $F_{\delta}$. By using the arguments in the proof of Theorem 4.1 in [BBGZ], we can further show that $\phi_{\delta}$ is a critical point of $F_{\delta}$, so it satisfies (3) for some $\lambda$.

Next, we estimate $\lambda$. From (5), we know that

$$
\int_{M}\left|\phi_{j}\right| \omega_{0}^{n} \leq C\left(A, B, F_{\delta}(0), V\right) .
$$

It follows that

$$
\left|\left\{e^{-\phi_{j}} \geq C_{1}\right\}\right|=\left|\left\{\phi_{j} \leq-\ln C_{1}\right\}\right| \leq \frac{\left|\int_{M} \phi_{j} \omega_{0}^{n}\right|}{\ln C_{1}} \leq \frac{C\left(A, B, F_{\delta}(0), V\right)}{\ln C_{1}} .
$$

So we can choose $C_{1}>0$ such that

$$
\left|\left\{e^{-\phi_{j}} \geq C_{1}\right\}\right| \leq \frac{V}{4} .
$$

We also choose $\epsilon>0$ such that

$$
\left|\left\{\left|S_{i}\right|_{h_{i}}^{2} \leq \epsilon\right\}\right| \leq \frac{V}{4 k}, \text { where } i=1, \cdots, k .
$$

Put

$$
N=\left\{e^{-\phi_{j}} \leq C_{1}\right\} \cap\left(\cap_{i=1}^{k}\left\{\left|S_{i}\right|_{h_{i}}^{2} \geq \epsilon\right\}\right),
$$

then

$$
|N| \geq \frac{V}{2} .
$$

On $N$, there is a $\delta_{0}=\delta_{0}\left(M, \omega_{0}, \Omega,\left\{S_{i}\right\},\left\{h_{i}\right\}\right)$ such that for any $\delta \leq \delta_{0}$ and each $i=1, \cdots, k$, we have

$$
e^{-C\left(A, B, F_{\delta}(0)\right)} \leq e^{-\phi_{j}} \leq C_{1}
$$

and

$$
\left(\left|S_{i}\right|_{h_{i}}^{2}+\delta e^{\frac{1-K_{i}}{1-\beta^{i}} \phi_{j}}\right)^{-\left(1-\beta^{i}\right)} \geq \frac{1}{2}\left|S_{i}\right|_{h_{i}}^{-2\left(1-\beta^{i}\right)} .
$$

So we get

$$
\int_{N} e^{-\phi_{\delta}} \prod_{i=1}^{k}\left(\left|S_{i}\right|_{h_{i}}^{2}+\delta e^{-\frac{1-K_{i}}{1-\beta^{i}} \phi_{\delta}}\right)^{-\left(1-\beta^{i}\right)} \Omega \geq C_{2}\left(M, \omega_{0}, \Omega,\left\{S_{i}\right\},\left\{h_{i}\right\}\right),
$$

and consequently

$$
\lambda \leq \frac{V}{C_{2}\left(M, \omega_{0}, \Omega,\left\{S_{i}\right\},\left\{h_{i}\right\}\right)} .
$$

On the other hand, we have

$$
e^{-\phi_{\delta}} \prod_{i=1}^{k}\left(\left|S_{i}\right|_{h_{i}}^{2}+\delta e^{-\frac{1-K_{i}}{1-\beta^{l}} \phi_{\delta}}\right)^{-\left(1-\beta^{i}\right)} \leq h\left(e^{-\phi_{\delta}}\right),
$$


we have

$$
\int_{M} e^{-\phi_{\delta}} \prod_{i=1}^{k}\left(\left|S_{i}\right|_{h_{i}}^{2}+\delta e^{-\frac{1-K_{i}}{1-\beta^{i}} \phi_{\delta}}\right)^{-\left(1-\beta^{i}\right)} \Omega \leq \int_{M} h\left(e^{-\phi_{\delta}}\right) \Omega=\int_{M} h(1) \Omega .
$$

Hence,

$$
\lambda \geq \frac{1}{V} \int_{M} h(1) \Omega .
$$

This completes the proof of our proposition.

Before we show the regularity of the solution $\phi_{\delta}$, let us first calculate the Ricci curvature of $\omega_{\delta}$.

Lemma 2.5. Assuming that $\omega_{\delta}$ is a smooth metric, then the Ricci curvature of $\omega_{\delta}$ satisfies

$$
\operatorname{Ric}\left(\omega_{\delta}\right) \geq K \omega_{\delta}
$$

Proof. Write (3) as:

$$
\left(\omega_{0}+\sqrt{-1} \partial \bar{\partial} \phi_{\delta}\right)^{n}=\lambda e^{\left(\Sigma\left(1-K_{i}\right)-1\right) \phi_{\delta}} \prod_{i=1}^{k}\left(\left|S_{i}\right|_{h_{i}}^{2} e^{\frac{1-K_{i}}{1-\beta^{i}} \phi_{\delta}}+\delta\right)^{-\left(1-\beta_{i}\right)} \Omega .
$$

Set

Clearly, we have

$$
h_{i}^{\delta}=h_{i} e^{\frac{1-K_{i}}{1-\beta^{l}} \phi_{\delta}} .
$$

$$
R\left(h_{i}^{\delta}\right)=R\left(h_{i}\right)-\frac{1-K_{i}}{1-\beta^{i}} \sqrt{-1} \partial \bar{\partial} \phi_{\delta} .
$$

Then the Ricci curvature $\operatorname{Ric}\left(\omega_{\delta}\right)$ is equal to

$$
\begin{aligned}
& \sqrt{-1}\left(\left(1+\sum_{i=1}^{k}\left(K_{i}-1\right)\right) \partial \bar{\partial} \phi_{\delta}+\sum_{i=1}^{k}\left(1-\beta^{i}\right) \partial \bar{\partial} \log \left(\left|S_{i}\right|_{h_{i}^{\delta}}^{2}+\delta\right)\right)+\operatorname{Ric}(\Omega) \\
\geq & \left(1+\sum_{i=1}^{k}\left(K_{i}-1\right)\right) \sqrt{-1} \partial \bar{\partial} \phi_{\delta}-\sum_{i=1}^{k}\left(1-\beta^{i}\right) \frac{\left|S_{i}\right|_{h_{i}^{\delta}}^{2} R\left(h_{i}^{\delta}\right)}{\left|S_{i}\right|_{h_{i}^{\delta}}^{2}+\delta}+\operatorname{Ric}(\Omega) \\
= & \left(1+\sum_{i=1}^{k}\left(K_{i}-1\right)\right) \sqrt{-1} \partial \bar{\partial} \phi_{\delta}-\sum_{i=1}^{k}\left(1-\beta^{i}\right) \frac{\left|S_{i}\right|_{h_{i}^{\delta}}^{2} R\left(h_{i}\right)}{\left|S_{i}\right|_{h_{i}^{\delta}}^{2}+\delta} \\
& +\sum_{i=1}^{k}\left(1-K_{i}\right) \frac{\left|s_{D_{i}}\right|_{h_{D_{i}}^{\phi_{\delta}}}^{2} \sqrt{-1} \partial \bar{\partial} \phi_{\delta}}{\left|s_{D_{i}}\right|_{h_{D_{i}}^{\phi_{\delta}}}^{2}+\delta}+\omega_{0}+\sum_{i=1}^{k}\left(1-\beta^{i}\right) R\left(h_{i}\right) \\
= & \sum_{i=1}^{k} \frac{\delta\left[\left(1-\beta^{i}\right) R\left(h_{i}\right)+\left(1-K_{i}\right) \omega_{0}\right]}{\left|S_{i}\right|_{h_{i}^{\delta}}^{2}+\delta}+\sum_{i=1}^{k}\left(K_{i}-1\right) \frac{\delta}{\left|S_{i}\right|_{h_{i}^{\delta}}^{2}+\delta} \omega_{\delta}+\omega_{\delta} \\
\geq & \left(\sum_{i=1}^{k}\left(K_{i}-1\right)+1\right) \omega_{\delta} .
\end{aligned}
$$


This lemma is proved.

Next we establish the regularity of the solution $\phi_{\delta}$.

Lemma 2.6. For some $\alpha=\alpha(M, \omega) \in(0,1)$, we have

$$
\left|\phi_{\delta}\right|_{C^{\alpha}\left(M, \omega_{0}\right)} \leq C_{3},
$$

where $C_{3}=C_{3}\left(M, \omega_{0}, \Omega,\left\{S_{i}\right\}, h_{0}\right)$.

Proof. From the above, we know that

$$
\phi_{\delta} \in \mathcal{K} \subset P S H_{\text {full }}\left(M, \omega_{0}\right),
$$

where $\mathcal{K}$ is a weak compact subset. So by [Zer], for any $p>1$, $\left|e^{-\phi_{\delta}}\right|_{L_{p}}$ is uniformly bounded by some constant $C_{p}$ depending on $\mathcal{K}$. For any $q \in\left(1, \min \left\{\frac{1}{1-\beta^{i}}\right\}\right)$, we have $\left|\prod_{i=1}^{k}\left(\left|S_{i}\right|_{h_{i}}^{2}+\delta e^{\frac{1-K_{i}}{1-\beta^{i}} \phi_{\delta}}\right)^{-\left(1-\beta^{i}\right)}\right|_{L^{q}} \leq\left.\left.\left|\prod_{i=1}^{k}\right| S_{i}\right|_{h_{i}} ^{-2\left(1-\beta^{i}\right)}\right|_{L^{q}} \leq C\left(M, \omega_{0}, \Omega,\left\{S_{i}\right\},\left\{h_{i}\right\}, q\right)$.

It follows from the Hölder inequality that for any $p \in\left(1, \min \left\{\frac{1}{1-\beta^{i}}\right\}\right)$ and some constant $C$ independent of $\delta$,

$$
\left|e^{-\phi_{\delta}} \prod_{i=1}^{k}\left(\left|S_{i}\right|_{h_{i}}^{2}+\delta e^{\frac{1-K_{i}}{1-\beta^{i}} \phi_{\delta}}\right)^{-\left(1-\beta^{i}\right)}\right|_{L_{p}} \leq C
$$

So the lemma follows.

Proposition 2.7. There exists $\delta_{l} \rightarrow 0$ such that $\phi_{\delta_{l}}$ converges to $\phi_{K E}+c$ in the $C^{0}$-topology for some constant $c$.

Proof. From the above lemma, we can choose a subsequence $\phi_{\delta_{l}}$ which converges to a continuous function $\psi$, moreover, for some $\lambda, \psi$ satisfies

$$
\left(\omega_{0}+\sqrt{-1} \partial \bar{\partial} \psi\right)^{n}=\lambda e^{-\psi} \prod_{i=1}^{k}\left|S_{i}\right|_{h_{i}}^{-2\left(1-\beta^{i}\right)} \Omega .
$$

By the uniqueness result of Berndtsson in [Bo], we know that $\psi=\phi_{K E}+c$.

Now we show that $\phi_{\delta}$ is a smooth function. We need a special case of Proposition 2.1 in [GP].

Proposition 2.8. Let $\phi$ be a solution of

$$
\omega_{\phi}^{n}=e^{\psi^{+}-\psi^{-}} \omega_{0}^{n},
$$

where $\omega_{\phi}=\omega_{0}+\sqrt{-1} \partial \bar{\partial} \phi$ and $\psi^{ \pm}$are smooth functions. We further assume that there exists $a C>0$ such that:

(i) $|\phi| \leq C$

(ii) $\left|\psi^{ \pm}\right| \leq C$ and $\sqrt{-1} \partial \bar{\partial} \psi^{ \pm} \geq-C \omega_{0}$;

(iii) The curvature tensor $R\left(\omega_{0}\right)$ of $\omega_{0}$ is bounded from below by $-C$. 
Then there exists a constant $A>0$ depending only on $C$ such that

$$
\frac{1}{A} \omega_{0} \leq \omega_{\phi} \leq A \omega_{0} .
$$

Choose a sequence of smooth $\omega_{0}$-psh functions $\psi_{j}$ which converges to $\phi_{\delta}$ in the $C^{0}$-norm. Note that by saying that $\psi_{j}$ is $\omega_{0}$-psh, we mean

$$
\omega_{0}+\sqrt{-1} \partial \bar{\partial} \psi_{j} \geq 0 .
$$

Lemma 2.9. If $\phi_{j}$ is any solution of

$$
\left(\omega_{0}+\sqrt{-1} \partial \bar{\partial} \phi_{j}\right)^{n}=\lambda e^{-K \psi_{j}} \prod_{i=1}^{k}\left(\left|S_{i}\right|_{h_{i}}^{2} e^{\frac{1-K_{i}}{1-\beta^{i}} \psi_{j}}+\delta\right)^{-\left(1-\beta^{i}\right)} \Omega,
$$

then for some $C=C\left(M, \delta,\left|\phi_{\delta}\right|_{C^{0}}\right)$,

$$
\left|\Delta \phi_{j}\right| \leq C
$$

Proof. Put

$$
u_{j}=\sum_{i=1}^{k}\left(1-\beta^{i}\right) \log \left(\left|S_{i}\right|_{h_{i}}^{2} e^{\frac{1-K_{i}}{1-\beta^{i}} \psi_{j}}+\delta\right) .
$$

For each $i$, we put for simplicity

$$
\left\|S_{i}\right\|_{j}^{2}=\left|S_{i}\right|_{h_{i}}^{2} e^{\frac{1-K_{i}}{1-\beta^{l}} \psi_{j}}
$$

Using the computation as above, we know that,

$$
\begin{aligned}
\sqrt{-1} \partial \bar{\partial} u_{j} & \geq-\sum_{i=1}^{k}\left(1-\beta^{i}\right) \frac{\left\|S_{i}\right\|_{j}^{2}}{\left\|S_{i}\right\|_{j}^{2}+\delta} R\left(h_{i}\right)+\sum_{i=1}^{k}\left(1-K_{i}\right) \frac{\left\|S_{i}\right\|_{j}^{2}}{\left\|S_{i}\right\|_{j}^{2}+\delta} \sqrt{-1} \partial \bar{\partial} \psi_{j} \\
& \geq \sum_{i=1}^{k} \frac{\delta}{\left\|S_{i}\right\|_{j}^{2}+\delta}\left(\left(1-\beta^{i}\right) R\left(h_{i}\right)+\left(1-K_{i}\right) \omega_{0}\right) \\
& -\sum_{i=1}^{k}\left(1-\beta^{i}\right) R\left(h_{i}\right)-(1-K) \omega_{0}
\end{aligned}
$$

Here we have used the fact:

$$
\omega_{0}+\sqrt{-1} \partial \bar{\partial} \psi_{j} \geq 0
$$

By our assumption,

$$
\left(1-\beta^{i}\right) R\left(h_{i}\right)+\left(1-K_{i}\right) \omega_{0} \geq 0,
$$

so for some $C>0$, we have

$$
\sqrt{-1} \partial \bar{\partial} u_{j} \geq-C \omega_{0}
$$

Since $K=\sum_{i=1}^{k}\left(K_{i}-1\right)+1 \leq 1$, we have

$$
\sqrt{-1} \partial \bar{\partial}\left((-K+1) \psi_{j}\right) \geq(K-1) \omega_{0} .
$$

The righthand side of (7) can be written as $e^{\psi_{+, j}-\psi_{-, j}}$, where

$$
\psi_{+, j}=(1-K) \psi_{j} \text {, and } \psi_{-, j}=u_{j}+\psi_{j} .
$$


It follows from the above that for some constant $C>0$, we have

$$
\sqrt{-1} \partial \bar{\partial} \psi_{ \pm, j} \geq-C \omega_{0}
$$

Hence, by Proposition 2.8, we have

$$
\left|\Delta \phi_{j}\right| \leq C_{\delta}
$$

where $C_{\delta}=C\left(M, \delta,\left|\phi_{\delta}\right|_{C^{0}}\right)$.

It follows from the uniqueness theorem for complex Monge-Ampere equations that $\phi_{i}$ converges to $\phi_{\delta}+c$ for some constant $c$, so we have

$$
\left|\phi_{\delta}\right|_{C^{1,1}\left(M, \omega_{0}\right)} \leq C_{\delta}
$$

By the Evans-Krylov theory, we know that for some $\alpha \in(0,1)$,

$$
\left|\phi_{\delta}\right|_{C^{2, \alpha}\left(M, \omega_{0}\right)} \leq C_{\delta}^{\prime},
$$

where $C_{\delta}^{\prime}$ may depend on $\delta$. Then, using bootstrap, we see that $\phi_{\delta}$ is a smooth function.

Now we derive uniform estimates of $\omega_{\delta}$ and prove the Gromov-Hausdorff convergence.

Lemma 2.10. There exists $C=C\left(M, \omega_{0},\left|\phi_{\delta}\right|_{C^{0}}\right)$ such that

$$
\frac{1}{C} \omega_{0} \leq \omega_{\delta} \leq C \prod_{i=1}^{k}\left|S_{i}\right|_{h_{i}}^{-2\left(1-\beta^{i}\right)} \omega_{0} .
$$

Proof. Since the Ricci curvature $\operatorname{Ric}\left(\omega_{\delta}\right)$ is bounded from below by $K \omega_{\delta}$, by the Chern-Lu inequality, we have

$$
\Delta_{\omega_{\delta}} \log \operatorname{tr}_{\omega_{\delta}} \omega_{0} \geq-K-B \operatorname{tr}_{\omega_{\delta}} \omega_{0},
$$

where $B$ is the upper bound of the bisectional curvature of $\omega_{0}$. Then we have

$$
\Delta_{\omega_{\delta}}\left(\log \operatorname{tr}_{\omega_{\delta}} \omega_{0}-(B+1) \phi_{\delta}\right) \geq \operatorname{tr}_{\omega_{\delta}} \omega_{0}-n(B-1)-K .
$$

So by the Maximum Principle, we get the lower bound of $\omega_{\delta}$. Then the upper bound follows from (3) and lower bound of $\omega_{\delta}$.

By the regularity theory on higher derivatives, $\phi_{\delta}$ converges to $\phi$ smoothly outside $|D|$. The following proposition is standard to experts in the field in view of what we had above. It can be proved by following the arguments in the proof of a similar result in [Ti15].

Proposition 2.11. For the sequence $\phi_{\delta_{i}}$ as in Proposition $2.7\left(M, \omega_{\delta_{i}}\right)$ converges to $(M, \omega)$ in the Gromov-Hausdorff sense.

Proof. Let us outline a proof here for the readers' convenience. Fix a ball $B_{p}(r, \omega) \subset$ $M \backslash D$. Since $\omega_{\delta_{i}}$ converges smoothly outside $D, B_{p}\left(r, \omega_{\delta_{i}}\right)$ converges to $B_{p}(r, \omega)$ smoothly. In particular, the volume of $B_{p}\left(r, \omega_{\delta_{i}}\right)$ has a definite lower bound. So $\left(M, \omega_{\delta_{i}}\right)$ is locally non-collapsing, and we can consider the pointed Gromov-Hausdorff convergence. Taking any convergent subsequence of $\left(M, \omega_{\delta_{i}}\right)$, denote the limit by 
$(X, d)$. Because $M \backslash \bigcup_{i=1}^{k} D_{i}$ is a convex dense subset of $M$, and $\omega_{\delta_{i}}$ converges to $\omega$ smoothly in this set, we will have a 1-Lipschitz map

$$
\iota:(M, \omega) \rightarrow(X, d),
$$

which is a local isometry on $M \backslash D$. By the volume convergence, $\iota(M \backslash D)$ is a full $\mathcal{H}^{n}$ measure subset of $X$. Then we know that $\iota(M \backslash D)$ is a open and dense subset in the regular part of $X$. By Theorem 3.7 in [CC2], $\iota(M \backslash D)$ is weakly convex, i.e. for any point $x \in \iota(M \backslash D)$, there is a subset $C_{x} \subset X$ of measure zero such that there is a minimal geodesic from $x$ to any point outside $C_{x}$. It follows that the induced distance on $\iota(M \backslash D)$ is equal to the intrinsic distance. So $\iota$ is an isometry.

\section{BASIC ESTIMATES FOR CONIC KäHLER-EINSTEIN METRICS}

Suppose that $\omega$ is a conic Kähler-Einstein metric on $M$ satisfying:

$$
\operatorname{Ric}(\omega)=t \omega+\sum_{i=1}^{k}\left(1-\beta^{i}\right) D_{i}, \omega \in 2 \pi c_{1}(L),
$$

where $L$ is $\mathbb{Q}$-line bundle and $t \in[\mu,+\infty)$ for some $\mu>0$. We are going to prove some basic estimates which will be used later. Denote the volume of $\omega$, which is a cohomological constant, by

$$
V=(2 \pi)^{n} \int_{M} c_{1}(L)^{n}
$$

Since $C_{0}^{\infty}\left(M \backslash \cup_{i=1}^{k} D_{i}\right)$ is dense in $H^{1}(M, \omega)$ and the Ricci curvature of $\omega$ is bounded from below by a positive constant, we have that the Sobolev constant $C_{s}$ of $\omega$ is uniformly bounded by a constant $C=C(n, \mu, V)$.

Now we give an application of the Moser iteration to conic Kähler-Einstein metrics.

Lemma 3.1. Assume that $f$ is a non-negative function satisfying:

$$
f \in L^{\infty}(M) \cap C^{\infty}\left(M \backslash \cup_{i=1}^{k} D_{i}\right)
$$

and for some positive constant $A \geq 1$,

$$
\Delta f \geq-A f, \text { in } M \backslash \cup_{i=1}^{k} D_{i} .
$$

Then we have

$$
|f|_{L^{\infty}(M)} \leq C A^{n}|f|_{L^{1}(M, \omega)},
$$

where $C$ is a constant depending on dimension $n$ and the Sobolev constant $C_{S}$ defined by: For any $f \in H^{1}(M, \omega)$, we have

$$
\left(\int_{M}|f|^{\frac{2 n}{n-1}} \omega^{n}\right)^{\frac{n-1}{n}} \leq C_{S}\left(\int_{M}|\nabla f|^{2} \omega^{n}+\int_{M}|f|^{2} \omega^{n}\right) .
$$

Proof. As before, we denote by $h_{i}$ a smooth Hermitian metric on the line bundle $L_{D_{i}}$ associated to $D_{i}$ and $S_{i}$ a defining section of $D_{i}$. Let $\tilde{\eta}$ be a cut-off function on $\mathbb{R}$ satisfying: $\tilde{\eta}(t)=1$ for $t \geq 2, \tilde{\eta}(t)=0$ for $t \leq 1$, and $\left|\tilde{\eta}^{\prime}\right| \leq 2$. 
Define

$$
\gamma_{\epsilon}=\prod_{i=1}^{k} \tilde{\eta}\left(\epsilon \log \left(-\log \left|S_{i}\right|_{h_{i}}^{2}\right)\right)
$$

Then $\gamma_{\epsilon}$ has its support in $M \backslash \cup_{i=1}^{k} D_{i}$ satisfying:

$$
\int_{M}\left|\nabla \gamma_{\epsilon}\right|^{2} \omega^{n} \leq C \epsilon
$$

where $C$ is a constant. Multiplying both sides of (8) by $\gamma_{\epsilon}^{2} f^{p-1}$, we have

$$
-\gamma_{\epsilon}^{2} f^{p-1} \Delta f \leq A \gamma_{\epsilon}^{2} f^{p}
$$

Integrating by parts, we have

(10) $\frac{4(p-1)}{p^{2}} \int_{M} \gamma_{\epsilon}^{2}\left|\nabla f^{\frac{p}{2}}\right|^{2} \omega^{n}+2 \int_{M} \gamma_{\epsilon} f^{p-1}\left\langle\nabla f, \nabla \gamma_{\epsilon}\right\rangle \omega^{n} \leq A \int_{M} \gamma_{\epsilon}^{2} f^{p} \omega^{n}$.

Assume that $|f| \leq Q$, we have

$$
\begin{aligned}
\left|\int_{M} \gamma_{\epsilon} f^{p-1}\left\langle\nabla f, \nabla \gamma_{\epsilon}\right\rangle \omega^{n}\right| & \leq\left(\int_{M} \gamma_{\epsilon}^{2} f^{2 p-2}|\nabla f|^{2} \omega^{n}\right)^{\frac{1}{2}}\left(\int_{M}\left|\nabla \gamma_{\epsilon}\right|^{2} \omega^{n}\right)^{\frac{1}{2}} \\
& \leq Q^{\frac{p}{2}}\left(\int_{M} \gamma_{\epsilon}^{2} f^{p-2}|\nabla f|^{2} \omega^{n}\right)^{\frac{1}{2}}\left(\int_{M}\left|\nabla \gamma_{\epsilon}\right|^{2} \omega^{n}\right)^{\frac{1}{2}} \\
& \leq C Q^{\frac{p}{2}} \sqrt{\epsilon}\left(\int_{M} \gamma_{\epsilon}^{2} f^{p-2}|\nabla f|^{2} \omega^{n}\right)^{\frac{1}{2}} .
\end{aligned}
$$

Let $\epsilon \rightarrow 0$, from (10), we have

$$
\frac{4(p-1)}{p^{2}} \int_{M}\left|\nabla f^{\frac{p}{2}}\right|^{2} \omega^{n} \leq A \int_{M} f^{p} \omega^{n}
$$

Using the Sobolev inequality, we have

$$
\frac{4(p-1)}{p^{2}}\left[\frac{1}{C_{S}}\left(\int_{M} f^{\frac{n p}{n-1}} \omega^{n}\right)^{\frac{n-1}{n}}-\int_{M} f^{p} \omega^{n}\right] \leq A \int_{M} f^{p} \omega^{n} .
$$

Thus for $\tau=\frac{n}{n-1}$, we obtain

$$
|f|_{L^{p \tau}} \leq\left(A p C_{S}\right)^{\frac{1}{p}}|f|_{L^{p}}, p \geq 2 .
$$

Putting $p_{k}=2 \tau^{k-1}$, we get

$$
|f|_{L^{p_{k}}} \leq\left(A p_{k-1} C_{S}\right)^{\frac{1}{p_{k-1}}}|f|_{L^{p_{k-1}}} .
$$

Taking $k \rightarrow \infty$, we have

$$
|f|_{L^{\infty}} \leq A^{\frac{n}{2}} C|f|_{L^{2}},
$$

where $C=C\left(C_{S}, n\right)$ depends only on $C_{S}$ and $n$. Taking $p=2$ in (12), using the Hölder inequality

we have

$$
|f|_{L^{2}}^{\frac{n+1}{n}} \leq|f|_{L^{2 \gamma}}|f|_{L^{1}}^{\frac{1}{n}},
$$

$$
|f|_{L^{2}} \leq C A^{\frac{n}{2}}|f|_{L^{1}}
$$


The lemma is proved.

Since $L$ is $\mathbb{Q}$-line bundle, we can choose an integer $l_{0}$ such that $L^{l_{0}}$ is a line bundle and take a hermitian metric on $L^{l_{0}}$ such that

$$
R\left(h_{0}\right)=l_{0} \omega .
$$

In the sequel, we always take $l$-th power of $L$ for $l=m l_{0}$ being a multiple of $l_{0}$. So for simplicity, we can think that there is a hermitian metric $h$ on $L$ with its curvature $R(h)$ being $\omega$. Now we give the gradient estimate of holomorphic sections for conic metrics.

Lemma 3.2. For $s \in H^{0}\left(M, L^{l}\right)$, we have

$$
|s|_{h}+l^{-\frac{1}{2}}|\nabla s|_{\omega \otimes h} \leq C l^{\frac{n}{2}}\left(\int_{M}|s|^{2} \omega^{n}\right)^{\frac{1}{2}},
$$

where $C$ is again a constant depending only on $n$ and the Sobolev constant $C_{S}$ of $(M, \omega)$.

Proof. In $M \backslash \cup D_{i}$, it holds that

$$
\Delta|s|_{h}^{2} \geq-n l|s|_{h}^{2}
$$

and

$$
\Delta|\nabla s|_{\omega \otimes h}^{2} \geq-l(n+2)|\nabla s|_{\omega \otimes h}^{2} .
$$

Then (13) follows from Lemma 3.1 .

We also need Hörmander's $L^{2}$ estimate.

Lemma 3.3. For any $v \in C^{\infty}\left(\Gamma\left(M, L^{l}\right)\right)$, there exists a solution $\sigma \in C^{\infty}\left(\Gamma\left(M, L^{l}\right)\right)$ such that $\bar{\partial} \sigma=\bar{\partial} v$ with property:

$$
\int_{M}|\sigma|^{2} \omega^{n} \leq l^{-1} \int_{M}|\bar{\partial} v|^{2} \omega^{n}
$$

Proof. In $M \backslash \bigcup_{i=1}^{k} D_{i}$, we have the Weitzenböch formula

$$
\Delta_{\bar{\partial}} \theta=\bar{\nabla}^{*} \bar{\nabla} \theta+\omega(\theta, \cdot)+l \theta
$$

for $\theta \in \Lambda^{0,1}\left(L^{l}\right)$. Let $\gamma_{\epsilon}$ be the cut-off function supported in $M \backslash \bigcup_{i=1}^{k} D_{i}$ as above.

Multiplying both sides of the above identity by $\gamma_{\epsilon} \theta$ and integrating by parts, we have

$$
\int_{M} \gamma_{\epsilon}\left\langle\Delta_{\bar{\partial}} \theta, \theta\right\rangle \omega^{n}=\int_{M}\left(l \gamma_{\epsilon}|\theta|^{2}+\gamma_{\epsilon}\left(\omega \otimes h_{j}\right)(\theta, \theta)+\gamma_{\epsilon}|\bar{\nabla} \theta|^{2}-\left\langle\left(\bar{\nabla} \gamma_{\epsilon}, \bar{\nabla} \theta\right)_{\omega}, \theta\right\rangle_{h}\right) \omega^{n} .
$$

Taking $\epsilon \rightarrow 0$, noticing that

we get

$$
\int_{M}\left\langle\left(\bar{\nabla} \gamma_{\epsilon}, \bar{\nabla} \theta\right)_{\omega}, \theta\right\rangle_{h} \leq|\theta|_{L^{\infty}}|\bar{\nabla} \theta|_{L^{\infty}}\left(\int_{M}\left|\nabla \gamma_{\epsilon}\right|^{2} \omega_{j}^{n}\right)^{\frac{1}{2}} \rightarrow 0,
$$

$$
\int_{M}\left\langle\Delta_{\bar{\partial}} \theta, \theta\right\rangle \omega^{n} \geq l \int_{M}|\theta|^{2} \omega^{n}
$$


Since $\Lambda^{p, q}\left(L^{l}\right)$ is dense in $L^{2}\left(L^{l} \otimes \Omega^{p, q}\right)$, we know that $\Delta_{\bar{\partial}}$ is an invertible operator. Putting $\sigma=\bar{\partial}^{*} \Delta_{\bar{\partial}}^{-1} \bar{\partial} v$, the lemma is proved.

Like the eigenvalue estimate for smooth metrics, we have

Lemma 3.4. The first eigenvalue of $(M, \omega)$ is at least $t$.

Proof. By Theorem 2.1, there is a smooth approximation $\omega_{j}$ of $\omega$. The first eigenvalues $\lambda_{1}^{j}$ of $\omega_{j}$ are uniformly bounded and the first eigenfunctions $u_{j}$ are uniformly Lipschitz. By subsequence, $u_{j}$ converges to an eigenfunction $u$ on $(M, \omega)$ with eigenvalue

$$
\lambda=\lim _{j \rightarrow \infty} \lambda_{1}^{j}
$$

If $\lambda>\lambda_{1}$, we will have a function $v$ such that

$$
\int_{M}|\nabla v|^{2} \omega^{n}=\lambda_{1}, \quad \int_{M} v^{2} \omega^{n}=1
$$

It follows from the smooth convergence that

$$
\lim _{j \rightarrow \infty} \int_{M}|\nabla v|^{2} \omega_{j}^{n}=\lambda_{1}, \lim _{j \rightarrow \infty} \int_{M} v^{2} \omega_{j}^{n}=1 .
$$

So for any $\epsilon>0$, we can find an eigenfunction $v_{j}$ with eigenvalue not bigger than $\lambda_{1}+\epsilon$ when $j$ is large enough. It contradicts to (15). So we know that the first eigenfunction $u$ is the first eigenfunction. In particular, we have

$$
|\nabla u| \leq C,
$$

where $C=C\left(n, C_{S}\right)$ is some constant depending on only $n$ and $C_{S}$.

Now by the Weitzenböch formula, we have

$$
\Delta_{\bar{\partial}} \bar{\partial} u=\bar{\nabla}^{*} \bar{\nabla} \bar{\partial} u+\operatorname{Ric}(\bar{\partial} u,) .
$$

Let $\gamma_{\epsilon}$ be the cut-off function as above and multiply both sides of by $\gamma_{\epsilon}^{2} \bar{\partial} u$, we have

$$
\int_{M} \gamma_{\epsilon}^{2}\langle\Delta \bar{\partial} u, \bar{\partial} u\rangle \omega^{n}=\int_{M}\left\langle\bar{\nabla}^{*} \bar{\nabla} \bar{\partial} u, \gamma_{\epsilon}^{2} \bar{\partial} u\right\rangle \omega^{n}+t \int_{M} \gamma_{\epsilon}^{2}|\bar{\partial} u|^{2} \omega^{n} .
$$

Since $\omega$ is a conic Kähler-Einstein metric, Notice that

$$
\int_{M} \gamma_{\epsilon}^{2}\langle\Delta \bar{\partial} u, \bar{\partial} u\rangle \omega^{n}=\int_{M} \gamma_{\epsilon}^{2}\langle\bar{\partial} \Delta u, \bar{\partial} u\rangle \omega^{n}=\lambda_{1} \int_{M} \gamma_{\epsilon}^{2}|\bar{\partial} u|^{2} \omega^{n}
$$

Integration by parts, we have

$$
\int_{M}\left\langle\bar{\nabla}^{*} \bar{\nabla} \bar{\partial} u, \gamma_{\epsilon}^{2} \bar{\partial} u\right\rangle \omega^{n}=\int_{M} \gamma_{\epsilon}^{2}\langle\bar{\nabla} \bar{\partial} u, \bar{\nabla} \bar{\partial} u\rangle \omega^{n}+2 \int_{M}\left\langle\gamma_{\epsilon} \bar{\nabla} \bar{\partial} u, \bar{\nabla} \gamma_{\epsilon} \otimes \bar{\partial} u\right\rangle \omega^{n} .
$$

Since

$$
2\left|\int_{M}\left\langle\gamma_{\epsilon} \bar{\nabla} \bar{\partial} u, \bar{\nabla} \gamma_{\epsilon} \otimes \partial u\right\rangle \omega^{n}\right| \leq \eta \int_{M} \gamma_{\epsilon}^{2}\langle\bar{\nabla} \bar{\partial} u, \bar{\nabla} \bar{\partial} u\rangle \omega^{n}+\frac{C}{\eta} \int_{M}\left|\nabla \gamma_{\epsilon}\right|^{2} \omega^{n},
$$

we get

$$
(1-\eta) \int_{M} \gamma_{\epsilon}^{2}\langle\bar{\nabla} \bar{\partial} u, \bar{\nabla} \bar{\partial} u\rangle \omega^{n} \leq(\lambda-t) \int_{M} \gamma_{\epsilon}^{2}|\bar{\partial} u|^{2} \omega^{n}+\frac{C}{\eta} \int_{M}\left|\nabla \gamma_{\epsilon}\right|^{2} \omega^{n} .
$$


Taking $\epsilon \rightarrow 0$, and then $\eta \rightarrow 0$, we obtain

$$
\int_{M}\langle\bar{\nabla} \bar{\partial} u, \bar{\nabla} \bar{\partial} u\rangle \omega^{n} \leq\left(\lambda_{1}-t\right) \int_{M}|\bar{\partial} u|^{2} \omega^{n} .
$$

So $\lambda_{1} \geq t$.

\section{Compactness and Partial $C^{0}$-estimate}

Let $L$ be a $\mathbb{Q}$-line bundle on $M$. Define

$$
\mathcal{M}(K, A)=\left\{(M, \omega) \mid \omega \in 2 \pi c_{1}(L), \operatorname{Ric}(\omega) \geq K \omega, \operatorname{diam}(M, \omega) \leq A\right\} .
$$

The volume of any metrics in $\mathcal{M}(K, A)$ is a fixed constant $V$.

Let $D_{i}$ be the smooth divisors in $M$ as above and $\left(M, \omega_{j}, D\right)$, where $D$ denotes the divisor $\Sigma_{i=1}^{k}\left(1-\beta_{j}^{i}\right) D_{i}$, be a sequence of conic Kähler-Einstein metrics satisfying:

$$
\operatorname{Ric}\left(\omega_{j}\right)=t_{j} \omega_{j}+2 \pi \Sigma_{i=1}^{k}\left(1-\beta_{j}^{i}\right) D_{i}, \omega_{j} \in 2 \pi c_{1}(L),
$$

where both $t_{j}$ and $\beta_{j}^{i}$ are in $[\mu, 1]$ for some $\mu>0$. Assume that $\left(M, \omega_{j}, D\right)$ converges to a metric space $(X, d)$ in the Gromov-Hausdorff topology. From Theorem 2.1, we know that

$$
(X, d) \in \overline{\mathcal{M}}\left(K, \frac{\pi}{\sqrt{\mu}}\right) .
$$

We want to show that the limit is a normal variety. The key is to establish the partial $C^{0}$ estimate for $\omega_{j}$. Let us make it more precise. For any conic Kähler metric $\omega$, choose a hermitian metric $h$ on $L$ such that $\mathrm{R}(h)=\omega$. Then for a large integer $\ell$ such that $L^{\ell}$ is a line bundle, we have the $L^{2}$ metric on $H^{0}\left(M, L^{\ell}\right)$ :

$$
\left\langle s_{1}, s_{2}\right\rangle=\int_{M}\left\langle s_{1}, s_{2}\right\rangle_{h^{\otimes \ell}} \omega^{n}, s_{i} \in H^{0}\left(M, L^{\ell}\right) .
$$

Definition 4.1. For any orthonormal basis $\left\{s_{i}\right\}_{0 \leq i \leq N}$ of $H^{0}\left(M, L^{\ell}\right)$, we define a Bergman kernel as

$$
\rho_{\ell}(M, \omega)(x)=\Sigma_{i=0}^{N}\left|s_{i}\right|_{h^{\otimes \ell}}^{2}(x) .
$$

The partial $C^{0}$ estimate we want to establish for $\left(M, \omega_{j}\right)$ is stated as follows: There exists some large integer $\ell$ and some positive constant $c>0$, which may depend on $l$, such that

$$
\rho_{\ell}\left(M, \omega_{j}\right) \geq c .
$$

We will follow the strategy in [Ti15] to prove this.

At first, the Cheeger-Colding theory ([CC1]) assures the existence of tangent cones $C_{x}=C_{x}(X, d)$ as a metric cone, which may not be unique, at any $x \in X$. Then we have a decomposition

$$
X=\mathcal{R} \cup \mathcal{S},
$$

where $\mathcal{R}$ consists of all $x \in X$ which has a tangent cone $C_{x}$ isometric to the Euclidean space $\mathbb{R}^{2 n}$ and $\mathcal{S}$ is the complement, referred as the singular set. Also every tangent cone has a similar decomposition. Furthermore, it follows from [CC1] and [CCT] 
Lemma 4.2. There is a stratification of the singular set $\mathcal{S}$ of $X$ :

$$
\mathcal{S}=\bigcup_{k \geq 1} \mathcal{S}_{2 n-2 k}
$$

where $\mathcal{S}_{2 n-2 k}$ consists of all $x$ for which at least one tangent cone splits an Euclidean factor $\mathbb{C}^{n-k}$ and no tangent cones split Euclidean factor of dimension greater than $2 n-2 k$. We further have

$$
\operatorname{dim} \mathcal{S}_{2 n-2 k} \leq 2 n-2 k .
$$

Also every tangent cone $C_{x}$ of $X$ has the stratification of the same property.

It follows from volume convergence in $[\mathrm{Co}]$ and Anderson's result in $[\overline{\mathrm{An}}]$.

Lemma 4.3. If there exists $\bar{\beta}<1$ such that $\beta_{j}^{i} \leq \bar{\beta}$, then the regular part $\mathcal{R}$ is open, and the distance is induced by a smooth metric $\omega_{\infty}$ on $\mathcal{R}$. Moreover, for any compact set $K \subset \mathcal{R}$, there are $K_{j} \subset\left(M, \omega_{j}\right)$, such that $\left(K_{j}, \omega_{j}\right)$ converges to $\left(K, \omega_{\infty}\right)$ in the smooth topology.

For tangent cones, we have

Lemma 4.4. Assume that all $\beta_{j}^{i} \leq \bar{\beta}<1$. Let $C_{x}$ be a tangent cone of $(X, d)$ at $x$ :

$$
\left(C_{x}, \omega_{x}\right)=\lim _{a \rightarrow \infty}\left(X, r_{a}^{-1} d, x\right) .
$$

Then the regular part $\mathcal{R}\left(C_{x}\right)$ is open, and the distance is induced by a smooth metric $\omega_{x}$ on $\mathcal{R}\left(C_{x}\right)$. Moreover for any compact set $K \subset \mathcal{R}\left(C_{x}\right)$, there are $K_{j} \subset\left(\mathcal{R}, \omega_{\infty}\right)$, such that $\left(K_{a}, r_{a}^{-2} \omega_{\infty}\right)$ converges to $\left(K, \omega_{x}\right)$ in the smooth topology.

In general, there exists $l \in[1, k]$ so that $\beta_{j}^{i}(1 \leq i \leq l)$ approaches 1 and $\beta_{j}^{i}(l+1 \leq$ $i \leq k)$ is not greater than $\bar{\beta}$. From the volume comparison, there exists $\delta=\delta(n, \bar{\beta})$ such that for the metric $\omega_{j}$, if we have

$$
\operatorname{vol}\left(B_{r}\left(p_{j}, \omega_{j}\right)\right) \geq(1-\delta) V_{2 n} r^{2 n},
$$

where $B_{r}(p, \omega)$ denotes the metric ball of $(M, \omega)$ with radius $r$ and center $p$ and $V_{2 n}$ denotes the volume of the unit Euclidean ball, then

$$
B_{r}\left(p_{j}, \omega_{j}\right) \cap \bigcup_{i=l+1}^{k} D_{i}=\emptyset .
$$

We denote by $B_{r}\left(p_{\infty}, d\right)$ the limit of $B_{r}\left(p_{j}, \omega_{j}\right)$ in the Gromov-Hausdorff topology. We need the following regularity theorem for weak limits of conic Kähler-Einstein metrics (possibly singular).

Proposition 4.5. There exists $\delta=\delta(n, \bar{\beta}), \rho=\rho(n)$ such that if

$$
\operatorname{vol}\left(B_{r}\left(p_{\infty}, d\right)\right) \geq(1-\delta) V_{2 n} r^{2 n},
$$

then $B_{\rho r}\left(p_{\infty}, d\right)$ is biholomorphic to an open subset in $\mathbb{C}^{n}$ and the metric $d$ on $B_{\rho r}\left(p_{\infty}, d\right)$ is induced by a smooth Kähler-Einstein metric $\omega_{\infty}$, moreover, the GromovHausdorff limits $D_{i}^{\infty}$ of $D_{i}(1 \leq i \leq l)$ are divisors in $B_{\rho r}\left(p_{\infty}, d\right)$. 
Since we only know a prior that $\omega_{j}$ converges to $d$ in the weak topology, i.e., the Gromov-Hausdorff topology, we can not apply the Cheeger-Colding theorey directly to prove the smoothness of $B_{\rho r}\left(p_{\infty}, d\right)$ and $d$. There are two approaches to establishing such a regularity: one is to use the Ricci flow method as in [TW16] (also see Appendix B in [Ti15]), the other one works only in the complex case and is to use the technique of partial $C^{0}$-estimate. This second approach was first used in [CDS15] and will be used to prove Proposition 4.5. The first approach can be also applied after we extend certain results on the ordinary case of Ricci flow to the conic case.

Denote the scaled metrics

$$
\tilde{\omega}_{j}=r^{-2} \omega_{j} \text { and } \tilde{\omega}_{\infty}=r^{-2} \omega_{\infty} .
$$

We will denote by $\tilde{B}_{\rho}\left(p_{j}\right)$ and $\tilde{B}_{\rho}\left(p_{\infty}\right)$ the metric balls of radius $\rho$ in $\left(M, \omega_{j}\right)$ and $(X, \tilde{d})$, where $\tilde{d}=r^{-1} d$. Let $L_{E u c}$ be the trivial bundle over over the unit ball $B_{1}^{2 n}(0) \subset \mathbb{R}^{2 n}$ with curvature $\omega_{E u c}$ being the Kähler form of the Euclidean metric. Let $m(W)$ be the Minkowski measure of a subset $W$ with respect to a metric $\tilde{d}$, that is,

$$
m(W)=\inf \left\{A \mid \forall r>0, \exists A r^{2-2 n} \text { metric balls of radius } r \text { covering } W\right\} .
$$

Proposition 4.6. For any $A, \bar{\beta}>0$, there are $\rho=\rho(A), \delta=\delta(A, \bar{\beta})>0$ with the following property. First Assume

$$
\operatorname{vol}\left(\tilde{B}_{1}\left(p_{\infty}\right)\right) \geq(1-\delta) V_{2 n} .
$$

Next we assume that there is subset $W^{\infty} \subset \tilde{B}_{1}\left(p_{\infty}\right)$ with $m\left(W^{\infty}\right) \leq A$, such that $\left(\tilde{B}_{1}\left(p_{\infty}\right) \backslash W^{\infty}, \tilde{\omega}_{\infty}\right)$ is a smooth Kähler manifold and $D_{i}^{\infty}(1 \leq i \leq l)$ are divisors in $\tilde{B}_{1}\left(p_{\infty}\right) \backslash W^{\infty}$. Then for $j$ sufficiently large, we have

1. There is a local Kähler potential $\varphi_{j}$ for $\tilde{\omega}_{j}$ on the ball $\tilde{B}_{\rho}\left(p_{j}\right)$ such that

$$
\left|\varphi_{j}\right| \leq C(n) \rho^{2} .
$$

2. There is a holomorphic map $F_{j}: \tilde{B}_{\rho}\left(p_{j}\right) \mapsto \mathbb{C}^{n}$ which is a homeomorphism to its image and satisfies:

$$
\begin{gathered}
\left|\nabla F_{j}\right|_{\tilde{\omega}_{j}} \leq C(n) \\
B_{\frac{3 \rho}{4}}^{2 n}(0) \subset F_{j}\left(\tilde{B}_{\rho}\left(p_{j}\right)\right) \subset B_{\frac{5 \rho}{4}}^{2 n}(0) .
\end{gathered}
$$

By Anderson's result, we know that $\tilde{\omega}_{j}$ converges to $\tilde{\omega}_{\infty}$ in the smooth topology on any compact subset outside $W^{\infty}$ and $D_{i}^{\infty}$ for $i=1, \cdots, l$, moreover, for any $\epsilon_{1}, \eta>0$, there exists $\delta_{0}=\delta_{0}\left(\epsilon_{1}, \eta\right)$ such that if (18) holds for some $\delta \leq \delta_{0}$, there is injection

$$
\phi: \tilde{B}_{\frac{3}{4}}\left(p_{\infty}\right) \backslash T_{\epsilon_{1}}\left(W^{\infty}\right) \rightarrow B_{1}^{2 n}(0)
$$

such that

$$
\left|\phi^{*} \omega_{E u c}-\omega_{\infty}\right|_{C^{3}} \leq \eta,
$$

where $\omega_{E u c}$ denotes the Euclidean metric on $\mathbb{C}^{n}$. 
We denote by $L_{\infty}$ the limit of $L$ on $\tilde{B}_{\frac{1}{2}}\left(p_{\infty}\right) \backslash W^{\infty} \cup\left(\cup_{i=1}^{l} D_{i}^{\infty}\right)$. Since $\omega_{j}$ are conic Kähler-Einstein metrics with Kähler class $2 \pi c_{1}(L)$, they induce natural hermitian metrics on $L$ which converge to a hermitian metric on $L_{\infty}$ whose curvature is $\omega_{\infty}$. Moreover, since $\omega_{\infty}$ is smooth outside $W^{\infty}$, the line bundle $L_{\infty}$ can be extended to $\tilde{B}_{\frac{1}{2}}(0) \backslash W^{\infty}$. We start with the following lemma:

Lemma 4.7. Given any $\epsilon_{1}>0, \eta>0, A>0$, there exist $\tilde{\epsilon}_{1}, \tilde{\eta}, \delta_{1}>0$ such that if

$$
m\left(W^{\infty}\right) \leq A, \quad \operatorname{vol}\left(\tilde{B}_{1}\left(p_{\infty}\right) \geq\left(1-\delta_{1}\right) V_{2 n}\right.
$$

and for any circle $\gamma$ of radius $\tilde{\epsilon}_{1}$ in $\tilde{B}_{1}\left(p_{\infty}\right) \backslash T_{\tilde{\epsilon}_{1}}\left(W^{\infty}\right)$, we have

$$
\left|H_{\gamma}-I d\right| \leq \tilde{\eta},
$$

where $H_{\gamma}$ denotes the holonomy of $L_{\infty}^{\vee} \otimes \phi^{*} L_{E u c}$ around $\gamma$, there is a norm 1 section e of $L_{\infty}^{\vee} \otimes \phi^{*} L_{E u c}$ over $\tilde{B}_{1}\left(p_{\infty}\right) \backslash T_{\epsilon_{1}}\left(W^{\infty}\right)$ with $|D e| \leq \eta$.

Proof. This is a pretty trivial fact. For the readers' convenience, we include a standard proof by contradiction. One can also give an effective proof by estimating holonomy in terms of curvature. If Lemma is false, then for certain $\epsilon_{1}>0, \eta>$ $0, A>0$, we can find $\delta_{k} \mapsto 0, \eta_{i} \mapsto 0$ and $W_{i k} \subset \tilde{B}_{1}\left(p_{i k}\right)$ satisfying:

$$
m\left(W_{i k}\right) \leq A, \quad \operatorname{vol}\left(\tilde{B}_{1}\left(p_{i k}\right) \geq\left(1-\delta_{k}\right) V_{2 n}\right.
$$

and the holonomy of $L_{\infty}^{\vee} \otimes \phi^{*} L_{E u c}$ around any circle of radius $\frac{1}{i}$ inside $B_{1}(p i k) \backslash W_{i k}$ differs from $I d$ no more than $\eta_{i}$, but there is no norm 1 section $e$ with $|D e| \leq \eta$. Since $\omega_{\infty}$ can be approximated by smooth metrics with Ricci curvature bounded from below, by Letting $k$ go to $\infty$, we can get $W_{i}$ inside $B_{1}^{2 n}(0)$ with $m\left(W_{i}\right) \leq A$ and a flat bundle $L_{i}$ whose holonomy around any circle of radius $\frac{1}{i}$ inside $B_{1}^{2 n}(0) \backslash W_{i}$ is less than $\eta_{i}$. It follows that outside the limit $W^{\prime}$ of $W_{i}$ there is a flat bundle $L^{\prime}$. Also we have $m\left(W^{\prime}\right) \leq A$, so any loop $\gamma \subset B_{1}^{2 n}(0) \backslash W^{\prime}$ bounds a disc $\Sigma$ which intersects with $W^{\prime}$ at finitely many, say $\mathrm{m}$, points, consequently, $\gamma$ is homologous to $\mathrm{m}$ circles of arbitrarily small radius. By our choice of $W_{i}$, the holonomy of a circle in $B_{1}^{2 n}(0) \backslash W^{\prime}$ can be as close to $I d$ as one wants so long its radius is sufficiently small. Since $L^{\prime}$ is flat, it follows that the holonomy of $L^{\prime}$ around $\gamma$ is trivial. So $L^{\prime}$ must be a trivial bundle with a trivial connection. Then there is a norm 1 section $e_{i}$ of $L_{i}$ for $i$ sufficiently large such that $\left|D e_{i}\right| \leq \eta$. It is a contradiction. The lemma is proved.

Lemma 4.8. Given any $\tilde{\epsilon}_{1}>0$, there exists $\delta_{2}>0$, such that if

$$
\operatorname{vol}\left(\tilde{B}_{1}\left(p_{\infty}\right) \geq\left(1-\delta_{2}\right) V_{2 n},\right.
$$

then the holonomy of $L_{\infty}$ around any circle of radius $\tilde{\epsilon}_{1}$ in $\tilde{B}_{1}\left(p_{\infty}\right) \backslash W^{\infty}$ is less than $10 \tilde{\epsilon}_{1}^{2}$.

Proof. For a circle of radius $\tilde{\epsilon}_{1}$, after a slight perturbation we can assume that it has no intersection with $\bigcup_{i=1}^{l} D_{i}^{\infty}$. By the smooth convergence outside $W^{\infty} \bigcup_{i=1}^{l} D_{i}^{\infty}$, it suffices to estimate the holonomy for $L$ over $\tilde{B}_{1}\left(p_{j}\right)$. By the volume convergence result in [Co] and Appendix 1 in [CC1], for $\delta_{2}$ small, $\tilde{B}_{1}\left(p_{j}\right)$ is homeomorphic to $B_{1}^{2 n}(0)$ for $j$ sufficiently large. So the connection on $L$ is defined by a 1 -form $\varphi$. 
Pulling back the coordinates functions of $B_{1}^{2 n}(0)$ to $\tilde{B}_{1}\left(p_{j}\right)$, we can get the splitting functions $\left(f_{1}, f_{2}, \ldots, f_{2 n}\right)$ satisfying:

$$
\begin{aligned}
& \frac{1}{\operatorname{vol}\left(\tilde{B}_{1}\left(p_{j}\right)\right)} \int_{\tilde{B}_{1}\left(p_{j}\right)} \Sigma_{i}\left|\operatorname{Hess} f_{i}\right|^{2}+\Sigma_{i \neq j}\left|\left\langle\nabla f_{i}, \nabla f_{j}\right\rangle\right| \\
& +\frac{1}{\operatorname{vol}\left(\tilde{B}_{1}\left(p_{\infty}\right)\right)} \int_{\tilde{B}_{1}\left(p_{\infty}\right)} \Sigma_{i}\left(\left|\nabla f_{i}\right|-1\right)^{2}<\Psi\left(\delta_{2}\right) .
\end{aligned}
$$

As in $[\mathrm{CCT}]$, there are functions $\mathbf{r}$, $\mathbf{u}$ satisfying:

$$
\left\{\begin{array}{l}
\Delta \mathbf{r}^{2}=2 n \\
\left.\mathbf{r}^{2}\right|_{\partial \tilde{B}_{\frac{1}{2}}\left(p_{j}\right)}=\frac{1}{4}
\end{array}\right.
$$

and

$$
\left\{\begin{array}{l}
\Delta \mathbf{u}^{2}=2 n-2 \\
\left.\mathbf{u}^{2}\right|_{\partial \tilde{B}_{\frac{1}{2}}\left(p_{j}\right)}=\mathbf{r}^{2}-\sum_{j=1}^{2 n-2} f_{j}^{2} .
\end{array}\right.
$$

Denoting $\Phi=\left(f_{1}, f_{2}, \ldots, f_{2 n-2}\right)$ as in Theorem 2.63 in [CCT], we know that away from a subset of measure $\Psi(\delta)$, it holds that

$$
\int_{\Sigma_{z, \mathbf{u}}} \omega=\frac{u^{2}}{2}+\Psi\left(\delta_{2}\right)
$$

where $\Sigma_{z, u}=\Phi^{-1}(z) \cap\{\mathbf{u} \leq u\}$. For $\delta_{2}$ small, integrating by parts, we get

$$
\int_{\partial \Sigma_{z, \tilde{\epsilon}_{1}}} \varphi=\int_{\Sigma_{z, \tilde{\epsilon}_{1}}} d \varphi=\int_{\Sigma_{z, \tilde{\epsilon}_{1}}} \omega \leq 10 \tilde{\epsilon}_{1}^{2} .
$$

Lemma 4.9. For any $\epsilon_{1}, \eta>0$, there exists $\delta>0$ such that if

$$
\operatorname{vol}\left(\tilde{B}_{1}\left(p_{\infty}\right)\right) \geq(1-\delta) V_{2 n}
$$

then there is a hermitian bundle map $\psi$ from $L_{\infty}$ to $L_{E u c}$ over $\tilde{B}_{\frac{1}{2}}\left(p_{\infty}\right) \backslash T_{\epsilon_{1}}\left(W^{\infty}\right)$ lifting $\phi$ such that $|D \psi| \leq \eta$.

Proof. We can choose $\epsilon_{1}$ in Lemma4.7 such that $\tilde{\epsilon}_{1}^{2} \leq \tilde{\eta}$. Now by Lemma4.7 and Lemma 4.8, we only need to take $\delta=\min \left\{\delta_{1}, \delta_{2}\right\}$.

Now we are going to prove Proposition 4.6 .

Proof. Lemma 4.9 gives a bundle map $\psi: L_{\infty} \mapsto L_{E u c}$ over $\tilde{B}_{\frac{1}{2}}\left(p_{\infty}\right) \backslash T_{\epsilon_{1}}\left(W^{\infty}\right)$ lifting $\phi$ such that $|D \psi| \leq \eta$. Since $m\left(T^{\infty}\right) \leq A$, by using a well-known property of capacity, we get a cut-off function $\gamma_{\bar{\epsilon}}$ for any given $\bar{\epsilon}>0$ satisfying: Its support is contained in $T_{\epsilon_{1}}\left(W^{\infty}\right)$ for $\epsilon_{1}=\epsilon_{1}(\bar{\epsilon}, A)$ and its gradient has $L^{2}$ norm smaller than $\bar{\epsilon}$. We choose $\delta_{0}=\delta_{0}\left(\epsilon_{1}, \eta\right)$ such that (22) holds. As in the proof of the partial $C^{0}$ estimate in Section 5 in [Ti15], choosing $\bar{\epsilon}$ and $\eta=\eta(\bar{\epsilon})$ (also see the proof in Lemma 4.15), we can find a multiple $\ell=\ell(A)$ and holomorphic sections $s_{j}, s_{j}^{\alpha}$ of $L^{\ell}$ from approximate holomorphic sections

$$
\tau=\psi^{-1}\left(\gamma_{\bar{\epsilon}} e\right), \tau^{\alpha}=\psi^{-1}\left(\gamma_{\bar{\epsilon}} z^{\alpha}\right), \alpha=1, \cdots, n,
$$


where $e$ is the trivial section and $z^{\alpha}$ are the coordinates of $\mathbb{C}^{n}$. Then we define $\rho=\frac{1}{\ell}$ and the map $F_{j}$ by

$$
F_{j}=\left(\frac{s_{j}^{1}}{s_{j}}, \ldots, \frac{s_{j}^{n}}{s_{j}}\right)
$$

Now we use induction on the volume $\bar{A}=\sum_{i=1}^{l} \operatorname{vol}\left(D_{i} \cap \tilde{B}_{1}\left(p_{j}\right)\right)$ to prove Proposition 4.5. Define the volume density function by

$$
V(x, s)=r^{2-2 n} \sum_{i=1}^{l} \operatorname{vol}\left(D_{i} \cap B_{r}\left(x, \tilde{\omega}_{j}\right)\right) .
$$

Note that it depends on $\omega_{j}$ though we do not make it explicitly for simplicity.

We start with the following lemma:

Lemma 4.10. There are $\delta_{1}=\delta_{1}(n, \bar{\beta}), \kappa=\kappa(n)$ such that if

$$
\operatorname{vol}\left(\tilde{B}_{1}\left(p_{j}\right)\right) \geq\left(1-\delta_{1}\right) V_{2 n},
$$

then $V(q, \bar{r}) \geq \kappa$ for all $\bar{r}$ and $q \in \bigcup_{i=1}^{l} D_{i}$ such that $B_{\bar{r}}\left(q, \tilde{\omega}_{j}\right) \subset \tilde{B}_{\frac{1}{2}}\left(p_{j}\right)$.

Proof. Let $\delta=\delta\left(2^{2 n}, \bar{\beta}\right)$ as in Proposition 4.6. Let $\left(x_{j}, s_{j}\right)$ be the point in $\tilde{B}_{\frac{1}{2}}\left(p_{j}\right)$ attaining the minimum of $V(x, s)$ an $\bar{\omega}_{j}=s_{j}^{-2} \tilde{\omega}_{j}$. Clearly, for $B_{j}=B_{1}\left(x_{j}, \bar{\omega}_{j}\right)$, the geodesic ball with radius 1 of $\bar{\omega}_{j}$, we have

$$
m\left(B_{j} \bigcap \bigcup_{i=1}^{l} D_{i}\right) \leq 2^{2 n} .
$$

Let $B_{\infty}$ be the limit of $B_{j}$ and $W^{\infty}$ be $B_{\infty} \cap \bigcup_{i=1}^{l} D_{i}^{\infty}$, then $\bar{\omega}_{j}$ converges to $\bar{\omega}_{\infty}$ smoothly in $B_{1}\left(x_{\infty}, \bar{\omega}_{\infty}\right) \backslash W^{\infty}$, where $x_{\infty}=\lim x_{j}$. Applying Proposition 4.6. we get a holomorphic map $F_{j}$ with bounded image. Using the monotonicity of corresponding $V(x, s)$ in the Euclidean space, we have $V\left(x_{j}, s_{j}\right) \geq \kappa(n)$. The lemma is proved.

The following is a known consequence of the well-established regularity theory.

Lemma 4.11. For any $C, A>0$, suppose that

$$
\sum_{i=1}^{l} \operatorname{vol}\left(D_{i} \bigcap \tilde{B}_{1}\left(p_{j}\right)\right) \leq A
$$

and there is a holomorphic map $F_{j}: \tilde{B}_{1}\left(p_{j}\right) \rightarrow \mathbb{C}^{n}$ which is a homeomorphism to its image and satisfies

$$
\left(F_{j}\right)_{*} \omega_{j}=\sqrt{-1} \partial \bar{\partial} \varphi_{j} \text { with }\left|\varphi_{j}\right| \leq C \text { and }\left|\nabla F_{j}\right| \leq C .
$$

Then the limiting metric $\tilde{d}$ on $\tilde{B}_{\frac{1}{2}}\left(p_{\infty}\right)$ is induced by a smooth Kähler-Einstein metric $\omega_{\infty}$ and $D_{i}^{\infty}$ are divisors in $\tilde{\tilde{B}}_{\frac{1}{2}}\left(p_{\infty}\right)$. 
Proof. Using the holomorphic map $F_{j}$, we can write the following equations:

$$
\left(\sqrt{-1} \partial \bar{\partial} \varphi_{j}\right)^{n}=e^{-\varphi_{i}} \Pi_{i=1}^{l}\left|f_{i}\right|^{2\left(\beta_{j}^{i}-1\right)}|U|^{2},
$$

where $f_{i}$ is the defining function of $D_{i}$ and $U$ is a nonvanishing holomorphic function. Our assumptions say that for some constant $C$,

$$
\left|\varphi_{j}\right| \leq C, \quad \omega_{E u c} \leq C \sqrt{-1} \partial \bar{\partial} \varphi_{j} .
$$

Because the volume of $D_{i}$ is bounded with respect to any $\tilde{\omega}_{j}$, for each $i$, there is a limit $D_{i}^{\infty}$ of $D_{i}$. Furthermore, since $\beta_{j}^{i}$ tend to 1 , by taking a subsequence if necessary, $\varphi_{j}$ converge to a bounded $\varphi_{\infty}$ such that

$$
C^{-1} \omega_{E u c} \leq \sqrt{-1} \partial \bar{\partial} \varphi_{\infty} \leq C \omega_{E u c} .
$$

Then, it follows from the standard regularity theory for complex Monge-Ampere equations (see $[\mathrm{Bl}]$ ) that the limit $\omega_{\infty}$ is a smooth Kähler-Einstein metric.

Proof of Proposition 4.5. Choose $\delta$ to be $\delta_{1}$ in Lemma 4.10. If $\bar{A} \leq \kappa$, the proposition follows from Lemma 4.10 and Anderson's result. Now we assume that the proposition holds for $\bar{A} \leq 2^{m} \kappa$, we want to prove it still holds for $\bar{A} \leq 2^{m+1} \kappa$. Denote

$$
Z_{s}^{j}=\left\{q \in \tilde{B}_{\frac{1}{2}}\left(p_{j}\right) \mid V(q, s) \geq \frac{\bar{A}}{2}\right\}
$$

and

$$
W_{s}^{\infty}=\lim _{j \rightarrow \infty} Z_{s}^{j} \text { and } W^{\infty}=\bigcap_{s \leq 1} W_{s}^{\infty} .
$$

It follows from a standard covering argument that $m\left(W^{\infty}\right) \leq 2^{2 n}$. By induction, $\omega_{\infty}$ is smooth in $\tilde{B}_{\frac{1}{2}}\left(p_{\infty}\right) \backslash W^{\infty}$ and $D_{i}^{\infty}(1 \leq i \leq l)$ are divisors. Now by Proposition 4.6, there is a holomorphic map $F_{j}$ from $\tilde{B}_{\rho}\left(p_{j}\right)$ into $\mathbb{C}^{n}$ needed in Lemma 4.11, consequently, $\omega_{\infty}$ is smooth and $D_{i}^{\infty}(1 \leq i \leq l)$ are divisors in $\tilde{B}_{\frac{\rho}{2}}\left(p_{\infty}\right)$. The proposition is proved.

As a direct consequence of Proposition 4.5, we have the following regularity for the limit space $(X, d)$.

Lemma 4.12. The regular part $\mathcal{R}$ is open, and the distance function $d$ is induced by a smooth metric $\omega_{\infty}$ on $\mathcal{R}$ and $D_{i}^{\infty}$ are closures of divisors in $\mathcal{R}$. Moreover for any compact set $K \subset \mathcal{R} \backslash \bigcup_{i=1}^{l} D_{i}^{\infty}$, there are $K_{j} \subset\left(M, \omega_{j}\right)$, such that $\left(K_{j}, \omega_{j}\right)$ converges to $\left(K, \omega_{\infty}\right)$ in the smooth topology.

Similarly for the tangent cones, we have

Lemma 4.13. Let $C_{x}$ be any tangent cone of $(X, d)$ at $x$ given by

$$
\left(C_{x}, d_{x}\right)=\lim _{j \rightarrow \infty}\left(X, r_{j}^{-1} d, x\right),
$$

where $\left\{r_{j}\right\}$ is a sequence with $\lim r_{j}=0$. Then the regular part $\mathcal{R}\left(C_{x}\right)$ is open, and the distance $d_{x}$ is induced by a smooth metric $\omega_{x}$ on $\mathcal{R}\left(C_{x}\right)$. Also, $\left(X, r_{j}^{-1} d, x\right)$ converges smoothly to $\left(C_{x}, d_{x}\right)$ over $\mathcal{R}\left(C_{x}\right)$. 
The basic strategy to prove the partial $C^{0}$ estimate is to pull back a peak section from a tangent cone and then perturb it to a holomorphic section by solving the $\bar{\partial}$-equation.

On the regular part $\mathcal{R}$ of the limit $(X, d)$, we have a limit line bundle $L_{\mathcal{R}}$. On the regular part $\mathcal{R}\left(C_{x}\right)$ of any tangent cone $C_{x}$, we have

$$
\omega_{x}=\sqrt{-1} \partial \bar{\partial} \rho_{x}^{2}
$$

where $\rho_{x}$ is the distance to the vertex of $C_{x}$. Let $L_{0}$ be the trivial line bundle on $C_{x}$ equipped with the hermitian metric $e^{-\rho_{x}^{2}}$. We have the following lemma (Lemma 5.7 in [Ti15]).

Lemma 4.14. Let $C_{x}=\lim _{a \rightarrow \infty}\left(X, \sqrt{\ell_{a}} d, x\right)$ for $x \in(X, d)$, where $\ell_{a} \in \mathbb{Z}$ and $\ell_{a} \rightarrow$ $\infty$, and $K$ be any compact subset $\mathcal{R}\left(C_{x}\right)$. Then for any $\eta>0$, there exist positive integers $N=N(K, \eta)$ and $a_{0}$ such that for $a \geq a_{0}$, there exist $\ell=\ell(j) \leq N$ and $a$ sequence of $K_{a} \subseteq\left(\mathcal{R}, \omega_{\infty}\right)$ with property: There are diffeomorphisms $\phi_{a}: K \mapsto K_{a}$ and bundle homomorphisms $\psi_{a}$ lifting $\phi_{a}$ satisfying:

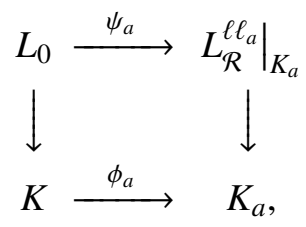

and

$$
\lim _{a \rightarrow \infty} \phi_{a}^{*}\left(\ell \ell_{a} \omega_{\infty}\right)=\omega_{x}, \quad\left|D \psi_{a}\right|_{\omega_{x}} \leq \eta \text { in } K
$$

where $|\cdot|_{\omega_{x}}$ is defined by $\omega_{\infty}, \omega_{x}$ and corresponding hermitian metrics on $L_{0}, L_{\mathcal{R}}$.

Let $\mathcal{S}_{x}$ denote the singular set of $C_{x}$, then we have

Lemma 4.15. Assume that for any $\bar{\epsilon}>0$, there is a smooth function $\gamma_{\bar{\epsilon}}$ on $B_{\bar{\epsilon}^{-1}}\left(o, \omega_{x}\right) \subset$ $C_{x}$ satisfying:

1) $\gamma_{\bar{\epsilon}} \equiv 0$ near $\mathcal{S}_{x}$ and $\gamma_{\bar{\epsilon}}(y) \equiv 1$ if $\operatorname{dist}\left(y, \mathcal{S}_{x}\right)>\bar{\epsilon}$;

2) $0 \leq \gamma_{\bar{\epsilon}} \leq 1$ and $\left|\nabla \gamma_{\bar{\epsilon}}\right| \leq C=C(\bar{\epsilon})$;

3) The integral bound:

$$
\int_{B_{\bar{\epsilon}^{-1}}(o)}\left|\nabla \gamma_{\bar{\epsilon}}\right|^{2} \omega_{x}^{n} \leq \bar{\epsilon}
$$

Then the partial $C^{0}$ holds at $x$, that is, there exists some large integer $\ell=\ell_{x}$ and some positive number $c_{x}$ such that for $j$ sufficiently large and $p \in M$ sufficiently close to $x$,

$$
\rho_{\ell}\left(M, \omega_{j}\right)(p) \geq c_{x} .
$$

Proof. Let $C=C\left(n, C_{s}\right)$ be the constant in (13). Choose a small $\delta \leq \frac{1}{8 C}$. Put

$$
V(x, \delta)=\left\{y \in C_{x} \mid \delta \leq \operatorname{dist}(y, x) \leq \delta^{-1}, \operatorname{dist}\left(y, \mathcal{S}_{x} \geq \delta\right\} .\right.
$$


We can choose $\delta$ smaller such that $V(x, 2 \delta)$ is not empty. Now in $V(x ; \delta)$, there exists $C_{1}$, which may depend on this region, such that if

$$
\bar{\partial} \sigma=f,
$$

we have the local estimate for $\sigma$ :

$$
|\sigma|_{C^{0}(V(x ; 2 \delta))} \leq C_{1}\left(|f|_{C^{0}(V(x ; \delta))}+\delta^{-2 n} \int_{V(x ; \delta)}|\sigma|^{2} \omega_{x}^{n}\right) .
$$

Choose $\bar{\epsilon}$ such that $100 C_{1} \bar{\epsilon} \leq \delta^{2 n+1}$, then by the assumption, we have $\gamma_{\bar{\epsilon}}$ with property: $\gamma_{\bar{\epsilon}} \equiv 1$ on $V(x, \delta)$. Let $\zeta$ be a cut-off function $\zeta$ such that $\zeta(t)=0$ for $t \geq 1$ and $\zeta(t)=1$ for $t \leq \frac{1}{2}$, then we set

$$
\beta(y)=\zeta\left(\frac{\bar{\epsilon}}{d(y, x)}\right) \zeta(\bar{\epsilon} d(y, x)) \gamma_{\bar{\epsilon}}(y), \quad \forall y \in C_{x} .
$$

Then, replacing $\gamma_{\bar{\epsilon}}$ by $\gamma_{\bar{\epsilon} / 2}$ if necessary, we can have

$$
\int_{C_{x}}|\nabla \beta|^{2} e^{-d^{2}(x,)} \omega_{x}^{n} \leq \bar{\epsilon} .
$$

Denote by $K$ the support of $\beta$. By Lemma 4.14, there are $K_{a} \subset \mathcal{R}$ converges to $K$ smoothly. Denoting by $e$ the constant section 1 of $L_{0}$, then by Lemma 4.14, we get a smooth section $\tau_{a}=\psi_{a}(\beta e)$ of $L_{\mathcal{R}}^{\ell \ell_{a}}$ over $\mathcal{R}$. For any $0<\eta \leq C_{1}^{-1} \delta$, if $a$ is sufficiently large, $\tau_{a}$ has the following properties:

$$
\left\{\begin{array}{l}
\left\|\tau_{a}\right\|_{C^{0}\left(\phi_{a}\left(V(x ; \delta) \cap B_{3 \delta}(x)\right)\right)}^{2} \geq(1-\eta) e^{-3 \delta^{2}} \\
\int_{X}\left|\tau_{a}\right|^{2} \omega_{\infty}^{n} \leq(1+\eta)\left(\frac{2 \pi}{\ell \ell_{a}}\right)^{n} \\
\left|\bar{\partial} \tau_{a}\right| \leq \eta \text { in } V(x ; \delta) ; \\
\int_{X}\left|\bar{\partial} \tau_{a}\right|^{2} \omega_{\infty}^{n} \leq(1+\eta) \frac{\bar{\epsilon}}{\left(\ell \ell_{a}\right)^{n-1}} .
\end{array}\right.
$$

By Lemma 4.12, $D_{i}^{\infty}(1 \leq i \leq l)$ are divisors in $\mathcal{R}$. Then we can choose a suitable cut-off function $\tilde{\gamma}_{\tilde{\epsilon}}$ supported outside $\bigcup_{i=1}^{l} D_{i}^{\infty}$ such that $\tilde{\tau}_{a}=\tau_{a} \tilde{\gamma}_{\tilde{\epsilon}}$ satisfies the above estimates with a slightly bigger $\eta$. By Lemma 4.12 , there are

$$
\phi_{j}: K_{a} \cap \operatorname{supp}\left(\tilde{\gamma}_{\tilde{\epsilon}}\right) \rightarrow\left(M, \omega_{j}\right)
$$

such that $\phi_{j}^{*} \omega_{j} \rightarrow \omega_{\infty}$, furthermore, these $\phi_{j}$ can be lifted to isomorphisms $\psi_{j}$ :

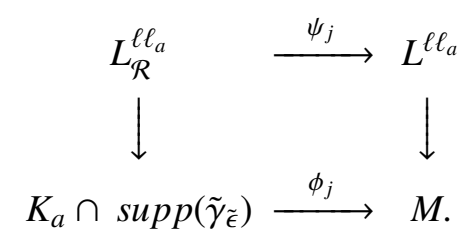

Then $v_{j}=\psi_{j}\left(\tilde{\tau}_{a}\right)$ is a smooth section of $L^{\ell \ell}$. For $j$ large enough, $v_{j}$ will also satisfy (28). By Lemma 3.3, we have a solution of

$$
\bar{\partial} \sigma_{j}=\bar{\partial} v_{j}
$$


satisfying

$$
\int_{M}\left|\sigma_{j}\right|_{h_{j}}^{2} \omega_{j}^{n} \leq(1+\eta) \frac{\bar{\epsilon}}{\left(\ell \ell_{a}\right)^{n}}
$$

where $h_{j}$ is the hermitian metric on $L^{\ell \ell_{a}}$ induced by $\omega_{j}$. Then $s_{j}=v_{j}-\sigma_{j}$ is a holomorphic section of $L^{\ell \ell_{a}}$. From (27), we have

$$
\left|\sigma_{j}\right|_{h_{j}}(q) \leq C_{1}\left(\eta+\frac{\bar{\epsilon}}{\delta^{2 n}}\right) \leq C_{1} \eta+\delta, \quad \forall q \in\left(\phi_{j} \circ \phi_{a}\right)(V(x ; 2 \delta)) .
$$

So

$$
\left|s_{j}\right| h_{j}(q) \geq 1-C_{1} \eta-\delta \geq 1-2 \delta .
$$

By the gradient estimate, we have

$$
\left|\nabla s_{j}\right|_{h_{j}}^{2} \leq C\left(n, C_{s}\right)\left(\ell \ell_{a}\right)^{n-1} \int_{M}|s|^{2} \omega_{j}^{n} \leq C\left(n, C_{s}\right)(1+\eta) \frac{1}{\ell \ell_{a}} .
$$

It follows that if $p$ is sufficiently close to $x$,

$$
\left|s_{j}\right|_{h_{j}}(p) \geq 1-2 \delta-3 C\left(n, C_{s}\right) \delta \geq \frac{1}{2} .
$$

The lemma is proved.

Now we have to show that for each tangent cone $C_{x}$, there is a cut-off function satisfying the conditions in Lemma 4.15. For the flat cone $\mathbb{C}_{\beta} \times \mathbb{C}^{n-1}$ with $\beta \leq \bar{\beta} \overline{1}$ we have the following cut-off function. Let $\bar{\delta} \in\left(0, \frac{1}{3}\right)$ and $\zeta$ be a cut-off function on $\mathbb{R}$ which satisfies $0 \leq \zeta \leq 1,\left|\zeta^{\prime}\right| \leq 1$ and

$$
\zeta=\left\{\begin{array}{l}
0, t>\log \left(-\log \bar{\delta}^{3}\right) \\
1, \quad t \leq \log (-\log \bar{\delta})
\end{array}\right.
$$

We define a cut-off function $\gamma_{\bar{\epsilon}}$ on $\mathbb{C}_{\beta} \times \mathbb{C}^{n-1}$ by

$$
\gamma_{\bar{\epsilon}}=\left\{\begin{array}{l}
1, \text { if } \rho(y)=\operatorname{dist}\left(y, S_{x}\right) \geq \bar{\epsilon} \\
\zeta\left(\log \left(-\log \left(\frac{\rho(y)}{\bar{\epsilon}}\right)\right)\right), \text { if } \rho(y) \leq \bar{\epsilon} .
\end{array}\right.
$$

Clearly, $\gamma_{\bar{\epsilon}} \equiv 1$ if $\rho(y) \geq \bar{\delta} \bar{\epsilon}$ and $\gamma_{\bar{\epsilon}} \equiv 0$ if $\rho(y) \leq \bar{\delta}^{3} \bar{\epsilon}$. Moreover, we have

$$
\operatorname{supp}\left|\nabla \gamma_{\bar{\epsilon}}\right| \subset \subset\left\{y \mid \bar{\delta}^{3} \bar{\epsilon} \leq \rho(y) \leq \bar{\delta} \bar{\epsilon}\right\} .
$$

Thus we have

$$
\begin{aligned}
& \int_{B_{\bar{\epsilon}^{-1}}(o)}\left|\nabla \gamma_{\bar{\epsilon}}\right|^{2} \omega_{x}^{n} \leq\left(\int_{\bar{\delta}^{3} \bar{\epsilon} \leq \rho(y) \leq \bar{\delta} \bar{\epsilon}}\left[\rho\left(-\log \left(\frac{\rho}{\bar{\epsilon}}\right)\right)\right]^{-2} \bar{\beta} \rho d \rho\right)\left(\int_{B_{\bar{\epsilon}^{-1}}(o)^{\prime}} \omega_{\text {flat }}^{n-1}\right) \\
& \leq \frac{2 \pi a_{n-1} \bar{\beta}}{\bar{\epsilon}^{2(n-1)}} \int_{\bar{\delta}^{3}}^{\bar{\delta}} \frac{d r}{r(\log r)^{2}} \\
& =\frac{2 \pi a_{n-1} \bar{\beta}}{\bar{\epsilon}^{2(n-1)}(-\log \bar{\delta})},
\end{aligned}
$$

\footnotetext{
${ }^{1} \bar{\beta}$ is the one given in Lemma 4.4
} 
where $B_{\bar{\epsilon}^{-1}}(o)^{\prime}$ denotes the $\bar{\epsilon}^{-1}$-ball in $\mathbb{C}^{n-1}$ centered at the origin and

$$
a_{n-1}=\frac{1}{(n-1) !} \operatorname{vol}\left(B_{1}\left(o, \omega_{\beta}\right)\right) \text {. }
$$

We also require the smooth convergence in the support of $\gamma_{\bar{\epsilon}}$. It will be done in the following lemma.

Now we can prove the local version of the partial $C^{0}$ estimate.

Lemma 4.16. Assume that for some sequence $\ell_{a} \rightarrow \infty$,

$$
C_{x}=\left(C_{x}, d_{x}\right)=\lim _{a \rightarrow \infty}\left(X, \sqrt{\ell_{a}} d, x\right)=\mathbb{C}_{\beta} \times \mathbb{C}^{n-1} .
$$

For any $\delta>0$, there exists $r_{x}>0$ and a Lipschitz map $F_{\infty}$ :

$$
F_{\infty}: B_{1}\left(x, r_{x}{ }^{-1} d\right) \rightarrow B_{2}\left(o, \omega_{\beta}\right)
$$

which is a $\delta$-Gromov-Hausdorff approximation. Moreover, $F_{\infty}\left(B_{1}\left(x, r_{x}^{-1} d\right) \cap \mathcal{S}\right)$ is a divisor.

Proof. Let $p_{j} \in M$ converge to $x$ in the Gromov-Hausdorff topology. By Lemma 4.15, for small $\delta>0$, there is an integer $\ell=\ell_{x}$ such that for $j$ large enough, we have holomorphic section $s_{j}$ satisfying

$$
\left.\left|s_{j}\right|\right|_{j}\left(p_{j}\right) \geq 1-\delta, \quad\left|\nabla s_{j}\right|_{h_{j}}^{2} \leq C\left(n, C_{s}\right)(1+\eta) \frac{1}{\ell \ell_{a}} .
$$

Putting $r_{x}=\frac{1}{2 C\left(n, C_{S}\right) \ell_{x}}$, we have

$$
\left|s_{j}\right|_{h_{j}}(q) \geq \frac{1}{2}-\delta \forall q \in B_{1}\left(p_{j}, r_{x}^{-1} \omega_{j}\right) .
$$

Now from smooth sections $\psi_{j}\left(\psi_{a}\left(z_{\alpha}\right)\right)(\alpha=1, \cdots, n)$, we can solve

$$
\bar{\partial} \sigma_{j}^{\alpha}=\bar{\partial} \psi_{j}\left(\psi_{a}\left(\gamma_{\bar{\epsilon}} z_{\alpha} e\right)\right)
$$

Putting $s_{j}^{\alpha}=\phi_{j}\left(\psi_{a}\left(\gamma_{\bar{\epsilon}} z_{\alpha} e\right)\right)-\sigma_{j}^{\alpha}$, we define

$$
F_{j}=\left(\frac{s_{j}^{1}}{s_{j}}, \ldots, \frac{s_{j}^{n}}{s_{j}}\right) .
$$

With respect to $r_{x}^{-1} \omega_{j}$, we have

$$
\left|\nabla F_{j}\right|_{r_{x}^{-1} \omega_{j}} \leq C\left(n, C_{S}\right) .
$$

Similar to (30), for any $q \in\left(\phi_{j} \circ \phi_{a}\right)(V(x ; 2 \delta))$ and $\eta, \bar{\epsilon}$ sufficiently small, we have

$$
\left|\frac{s_{j}^{1}}{s_{j}}-z_{\alpha} \circ\left(\phi_{j} \circ \phi_{a}\right)^{-1}\right|(q) \leq C_{1}\left(\eta+\frac{\bar{\epsilon}}{\delta^{2 n}}\right) \leq 2 \delta .
$$

This says that $F_{j}$ is a $2 \delta$-Gromov-Hausdorff approximation over $V(x, 2 \delta)$. By (34), we know that $F_{j}$ is a $6 C\left(n, C_{s}\right) \delta$-Gromov-Hausdorff approximation over $B_{1}\left(p_{j}, r_{x}^{-1} \omega_{j}\right)$ since $T_{2 \delta}(V(x, 2 \delta)) \supseteq B_{2}\left(o, \omega_{\beta}\right)$. Now $\bigcup_{i=l+1}^{k} F_{j}\left(D_{i} \cap B_{1}\left(p_{j}, r_{x}^{-1} \omega_{j}\right)\right)$ are divisors in $B_{2}\left(o, \omega_{\beta}\right)$ and converges to $F_{\infty}\left(B_{1}\left(x, r_{x}^{-1} d\right) \cap \mathcal{S}\right)$, where $F_{\infty}$ is the limit of $F_{j}$. Then the lemma is proved. 
Similarly, for the iterated tangent, we have

Lemma 4.17. Let $C_{x}=\lim _{a \rightarrow \infty}\left(X, \sqrt{\ell_{a}} d, x\right)$ be a tangent cone. For any $y \in$ $\mathcal{S}_{2 n-2}\left(C_{x}\right) \backslash \mathcal{S}_{2 n-4}\left(C_{x}\right)$ and any $\delta>0$, there is a small $s_{y}>0$ and a Lipschitz map $F_{\infty}$ from $B_{1}\left(x, s_{y}^{-2} \omega_{x}\right)$ to $B_{2}\left(o, \omega_{\beta}\right)$ which is a $\delta$-Gromov-Hausdorff approximation. Moreover, $F_{\infty}\left(B_{1}\left(y, s_{y}^{-2} \omega_{x}\right) \cap \mathcal{S}_{x}\right)$ is a divisor.

Proof. Since $y \in \mathcal{S}_{2 n-2}\left(C_{x}\right) \backslash \mathcal{S}_{2 n-4}\left(C_{x}\right)$, the iterated tangent cone $\left(C_{x}\right)_{y}$ at $y$ is isomorphic to $\mathbb{C}_{\beta} \times \mathbb{C}^{n-1}$. So we can do the same arguments on $\left(C_{x}\right)_{y}$ as we did in the proof of Lemma 4.16 to get the conclusion.

Proposition 4.18. There exists a positive integer $\ell$ and a positive number $c_{\ell}$ so that the partial $C^{0}$-estimate holds, i.e. $\rho_{\ell}\left(M, \omega_{j}\right) \geq c_{\ell}$.

Proof. By choosing a subsequence, we can assume that $\left(M, \omega_{j}\right)$ converges to a metric space $(X, d)$. To prove the Proposition, we only need to prove that for any $x \in X$ and $p_{j} \rightarrow x$, there exists a large integer $\ell=\ell_{x}$ and a positive number $c_{x}>0$ satisfying:

$$
\rho_{\ell}\left(M, \omega_{j}\right)(x) \geq c_{x} .
$$

It suffices to construct the required cut-off function on every tangent cone.

At first, by Lemma 4.17, for any $y \in \mathcal{S}_{2 n-2}\left(C_{x}\right) \backslash \mathcal{S}_{2 n-4}\left(C_{x}\right)$, we have a ball $B_{6 s_{y}}(y)$ with respect to $\omega_{x}$, such that $\mathcal{S}_{x} \cap B_{6 s_{y}}(y)$ is a local divisor, that is, there is a homeomorphism $F_{y}$ from $B_{6 s_{y}}(y)$ to a tangent cone $\left(C_{x}\right)_{y}$ such that $F_{y}\left(\mathcal{S}_{x} \cap B_{6 s_{y}}(y)\right)$ is a divisor. Put

$$
\hat{\mathcal{S}}_{x}=\mathcal{S}_{x} \backslash \bigcup_{y \in \mathcal{S}_{2 n-2}\left(C_{x}\right) \backslash \mathcal{S}_{2 n-4}\left(C_{x}\right)} B_{s_{y}}(y) .
$$

Clearly, it is a closed subset of $\mathcal{S}_{2 n-4}\left(C_{x}\right)$. Since the Hausdorff dimension of $\mathcal{S}_{2 n-4}\left(C_{x}\right)$ is strictly small than $2 n-3$, there is a finite cover of $\hat{\mathcal{S}}_{x} \cap B_{\bar{\epsilon}^{-1}}\left(o, \omega_{x}\right)$ by balls $B_{r_{a}}\left(q_{a}\right)$ such that

$$
\Sigma r_{\alpha}^{2 n-3} \leq \bar{\epsilon}
$$

So we have a cut-off function $\chi$ such that

$$
\chi \equiv 1 \text { in } \bigcup B_{r_{a}}\left(q_{a}\right), \quad \int_{C_{x}}|\nabla \chi|^{2} \omega_{x}^{n} \leq \bar{\epsilon} .
$$

We're going to construct a finite cover of $B_{\bar{\epsilon}^{-1}}\left(o, \omega_{x}\right) \backslash \bigcup B_{r_{a}}\left(q_{a}\right)$.

Now we cover $\mathcal{S}_{x} \cap\left(B_{\bar{\epsilon}^{-1}}\left(o, \omega_{x}\right) \backslash \bigcup B_{r_{\alpha}}\left(q_{\alpha}\right)\right)$ by finitely many balls $B_{s_{b}}\left(y_{b}\right)$. Let $\zeta$ be the cutoff function in (31). For each ball $B_{s_{b}}\left(y_{b}\right)$, we abbreviate $F_{b}=F_{y_{b}}$ and put

$$
\gamma_{\bar{\epsilon}, b}\left(x^{\prime}\right)=\zeta\left(\log \left(-\log \left(\frac{\left|f_{b}\right|\left(F_{b}\left(x^{\prime}\right)\right)}{\hat{\epsilon}}\right)\right)\right),
$$

where $\hat{\epsilon}=\hat{\epsilon}(\bar{\epsilon})$ and $f_{b}$ is the defining function of the divisor $F_{y}\left(\mathcal{S}_{x} \cap B_{6 s_{b}}\left(y_{b}\right)\right)$ in $B_{6 s_{b}}\left(y_{b}\right)$. For each $B_{6 s_{b}}\left(y_{b}\right)$, we choose a smooth function $\xi_{b}$ with support in $B_{1+\bar{\epsilon}^{-1}}\left(o, \omega_{x}\right)$ satisfying:

i) $0 \leq \xi_{b} \leq 1,\left|\nabla \xi_{b}\right| \leq s_{b}^{-1}$;

ii) $\operatorname{supp}\left(\xi_{b}\right) \subset B_{6 s_{b}}\left(y_{b}\right)$; 
iii) $\sum_{b} \xi_{b} \equiv 1$, on $\mathcal{S}_{x} \cap\left(B_{\bar{\epsilon}^{-1}}(o) \backslash \bigcup B_{r_{a}}\left(q_{a}\right)\right)$.

Then $\left\{\xi_{b}\right\}$ and $1-\sum_{b} \xi_{b}$ form a partition of unit for the cover $\left\{B_{6_{s}}\left(y_{b}\right), \cup B_{r_{a}}\left(q_{a}\right)\right\}$ on $B_{1+\bar{\epsilon}^{-1}}(o)$. Then we can patch these cut-off functions together to get

$$
\gamma_{\bar{\epsilon}}\left(x^{\prime}\right)=(1-\chi)\left(1-\sum \xi_{b}+\sum \xi_{b} \gamma_{\bar{\epsilon}, b}\left(x^{\prime}\right)\right) .
$$

As in the proof of Lemma 5.8 in [Ti15], if $\hat{\epsilon}$ is sufficiently small, we have

$$
\int_{B_{\bar{\epsilon}^{-1}}\left(o, \omega_{x}\right)}\left|\nabla \gamma_{\bar{\epsilon}}\right|^{2} \leq \bar{\epsilon}
$$

Since the metrics converges smoothly in $B_{\bar{\epsilon}^{-1}}\left(o, \omega_{x}\right) \backslash \cup B_{r_{\alpha}}\left(q_{\alpha}\right) \cup B_{s_{b}}\left(y_{b}\right)$, we get the cut-off function required.

Corollary 4.19. The Gromov-Hausodrff limit $(X, d)$ of $\left(M, \omega_{j}\right)$ is homeomorphic to a klt log Fano variety with a weak conic Kähler-Einstein metric.

Proof. By Proposition 4.18, we can embed $\left(M, \omega_{j}\right)$ into certain $\mathbb{C P}^{N}$ by using an orthonormal basis of $H^{0}\left(M, L^{\ell}\right)$ :

$$
\iota_{j}: M \rightarrow \mathbb{C P}^{N},
$$

moreover, $\iota_{j}$ is uniformly Lipschitz. Denote the Chow limit of $\iota_{j}\left(M, D_{1}, \ldots, D_{k}\right)$ by $\left(M_{\infty}, D_{1}^{\infty}, \ldots, D_{k}^{\infty}\right)$. Then we have a map:

$$
\iota_{\infty}:(X, d) \rightarrow M_{\infty}
$$

As showed in [DS][Ti15], $\iota_{\infty}$ is a homeomorphism and $M_{\infty}$ is a normal variety and $D_{i}^{\infty}(1 \leq i \leq k)$ are Weil divisors in $M_{\infty}$. Now we consider the metric $\left(\iota_{\infty}\right)_{*} \omega_{\infty}$ on $M_{\infty}$. Choose a local coordinate chart $\left(U, z_{\alpha}\right)$ around any smooth point $p \in M_{\infty}$ such that $O(1)$ is a trivial bundle and $D_{i}^{\infty} \cap U$ is defined by $f_{i}=0$. We can also regard $U$ as an open subset in $\mathbb{C}^{n}$. By the arguments in the proof of Lemma4.16, we can find holomorphic homeomorphisms

$$
F_{j}: V_{j} \subset M \rightarrow U \subset \mathbb{C}^{n}
$$

such that $\left(F_{j}\right)_{*} \omega_{j}$ converge to $\omega_{\infty}$ smoothly outside $\bigcup_{i=1}^{k} D_{i}^{\infty}$. Now we can write $\left(F_{j}\right)_{*} \omega_{j}=\sqrt{-1} \partial \bar{\partial} \varphi_{j}$ on $U$ such that for some $C>0$,

$$
\left|\varphi_{j}\right| \leq C, \quad \sqrt{-1} \partial \bar{\partial} \varphi_{j} \geq C^{-1} \omega_{E u c}
$$

Also, $\varphi_{j}$ satisfies

$$
\left(\sqrt{-1} \partial \bar{\partial} \varphi_{j}\right)^{n}=e^{-t_{j} \varphi_{j}} \Pi_{i=1}^{k}\left|f_{i}^{j}\right|^{2\left(\beta_{j}^{i}-1\right)}\left|\sigma_{j}\right|^{2}
$$

where $\sigma_{j}$ is a non-vanishing holomorphic section in $\Gamma\left(U,\left(F_{j}\right)_{*} K_{M}\right)$ and $f_{i}^{j}$ is a defining function of $F_{j}\left(D_{i}\right)$. Since $\beta_{j}^{i} \rightarrow 1(1 \leq i \leq l), \varphi_{j}$ converges to bounded function $\varphi_{\infty}$ on $U$ satisfying

$$
\left(\sqrt{-1} \partial \bar{\partial} \varphi_{\infty}\right)^{n}=e^{-t_{\infty} \phi_{\infty}} \Pi_{i=l+1}^{k}\left|f_{i}\right|^{2\left(\beta_{\infty}^{i}-1\right)}\left|\sigma_{\infty}\right|^{2},
$$


where $\sigma_{\infty}$ is a non-vanishing holomorphic section in $\Gamma\left(U, K_{M_{\infty}}\right)$. So $\omega_{\infty}$ is a weakly conic Kähler-Einstein metric. For any point $z \in M_{\infty}$, we can find a Zariski open subset $V$ so that (36) holds in the regular part of $V$. It is easy to see that

$$
\Pi_{i=l+1}^{k}\left|f_{i}\right|^{2\left(\beta_{\infty}^{i}-1\right)}\left|\sigma_{\infty}\right|^{2}
$$

is integrable over $V$. Thus, it follows that $\left(M_{\infty}, \sum_{i=l+1}^{k}\left(1-\beta_{\infty}^{i}\right) D_{i}^{\infty}\right)$ is a klt log pair.

\section{EXISTENCE OF CONIC KäHLER-EINSTEIN METRICS}

In this section, we will prove our main theorem. First, we recall the definition of $\log K$-polystable.

Definition 5.1. A normal test configuration $(X, \mathcal{D}, \mathcal{L})$ for $(M, D, L)$, where $D=$ $\sum_{i=1}^{k}\left(1-\beta^{i}\right) D_{i}$, consists of:

i) a normal log-pair $(X, \mathcal{D})$ with a $C^{*}$-equivariant morphism $\pi:(X, \mathcal{D}) \rightarrow \mathbb{C}$,

ii) $\mathcal{L}$ is an equivariant $\pi$-ample $\mathbb{Q}$-line bundle such that

$$
\left(\mathcal{X}_{t}, \mathcal{D}_{t}, \mathcal{L}_{t}\right) \cong(M, D, L), \quad \forall t \neq 0 .
$$

Let $(\bar{X}, \overline{\mathcal{D}}, \overline{\mathcal{L}})$ be the natural equivariant compactification of $(X, \mathcal{D}, \mathcal{L})$. The logCM weight is defined by

$$
w_{\text {lcm }}(X, \mathcal{D}, \mathcal{L})=\frac{n \overline{\mathcal{L}}^{n+1}+(n+1) \overline{\mathcal{L}}^{n}\left(K_{\bar{X} / \mathbb{C P}^{1}}+\overline{\mathcal{D}}\right)}{(n+1) L^{n}} .
$$

We say $(M, D, L) \log K$-semistable if $w_{\text {lcm }}(X, \mathcal{D}, \mathcal{L}) \geq 0$ for any normal test configuration $(X, \mathcal{D}, \mathcal{L})$.

We say $(M, D, L) \log K$-polystable if $w_{\text {lcm }}(X, \mathcal{D}, \mathcal{L}) \geq 0$ for any normal test configuration $(X, \mathcal{D}, \mathcal{L})$, and the equality holds if and only if $(X, \mathcal{D}, \mathcal{L}) \cong(X, D, L) \times \mathbb{C}$.

If the central fiber is a klt $\log$ Fano variety $\left(M_{0}, \Sigma_{i=1}^{k}\left(1-\beta^{i}\right) D_{i}^{0}, L_{0}\right)$, we have an embedding of $M_{0}$ into $\mathbb{C P}^{N}$ using $H^{0}\left(M_{0}, L^{\ell}\right)$ for a large $\ell$. Let $\bar{\omega}_{0}$ be the restriction of the Fubini-Study metric:

$$
\bar{\omega}_{0}=\frac{1}{\ell} \iota^{*} \omega_{F S}, \text { where } \iota: M_{0} \rightarrow \mathbb{C P}^{N} .
$$

Then as in [DT], we have

$$
w_{l c m}(X, \mathcal{D}, \mathcal{L})=-\int_{M_{0}} \theta_{X}\left(\operatorname{Ric}\left(\bar{\omega}_{0}\right)-\bar{\omega}_{0}\right) \wedge \bar{\omega}_{0}^{n-1}+\Sigma_{i=1}^{k}\left(1-\beta^{i}\right) \int_{D_{i}^{0}} \theta_{X} \bar{\omega}_{0}^{n-1},
$$

where $X$ generates the $\mathbb{C}^{*}$-action on $M_{0}$ and $\theta_{X}$ is the potential of $X$. As before, we will denote $\Sigma_{i=1}^{k}\left(1-\beta^{i}\right) D_{i}^{0}$ by $(1-\beta) D^{0}$ and the righthand side above by $\mathrm{Fut}_{\left(M_{0},(1-\beta) D^{0}\right)}(X)$.

Now we prove our main theorem.

Theorem 5.2. If $(M,(1-\beta) D)$ is log K-polystable, then there exists a conic KählerEinstein metric. 
Proof. Choose a large integer $\lambda$ large enough such that there is a smooth divisor $E \in|\lambda L|$. Fix a hermitian metric $h_{E}$ on $\lambda L$ such that

$$
R\left(h_{E}\right)=\lambda \omega_{0} \text {. }
$$

Consider the following continuity method through conic Kähler-Einstein metrics:

$$
\operatorname{Ric}\left(\omega_{t}\right)=t \omega_{t}+\frac{1-t}{\lambda}[E]+(1-\beta)[D] .
$$

It is equivalent to the following complex Monge-Ampére equation:

$$
\left(\omega_{0}+\sqrt{-1} \partial \bar{\partial} \phi_{t}\right)^{n}=e^{-t \phi_{t}}\left|s_{E}\right|_{h_{E}}^{-2 \frac{1-t}{\lambda}}\left|s_{D}\right|_{h_{D}}^{-2(1-\beta)} \Omega
$$

where $\Omega$ is the volume form given by

$$
\operatorname{Ric}(\Omega)=\omega_{0}+(1-\beta) R\left(h_{D}\right), \quad \int_{M} \Omega=\int_{M} \omega_{0}^{n}=V .
$$

The corresponding Lagrangian, also often referred as Ding functional, is

$$
F_{t}=J(\phi)-\int_{M} \phi \omega_{0}^{n}-\frac{1}{t} \log \left(\frac{1}{V} \int_{M} e^{-t \phi}\left|s_{E}\right|_{h_{E}}^{-2 \frac{1-t}{\lambda}}\left|s_{D}\right|_{h_{D}}^{-2(1-\beta)} \Omega\right) .
$$

Lemma 5.3. There exists a positive number $t_{0}=t_{0}(M, \lambda)$ such that for $t \in\left(0, t_{0}\right)$, $F_{t}$ is coercive.

Proof. For $\phi \in \operatorname{PS} H\left(M, \omega_{0}\right)$ with $\sup \phi=0$, we have

$$
\int_{M} e^{-\delta \phi} \Omega \leq A
$$

for some $\delta \leq \delta_{0}=\delta_{0}\left(M, \omega_{0}\right)$. We may assume that $\lambda \geq 2$, then there is $p>1$ such that

$$
\left(\int_{M}\left[\left|s_{E}\right|_{h_{E}}^{-2 \frac{1-t}{\lambda}}\left|s_{D}\right|_{h_{D}}^{-2(1-\beta)}\right]^{p} \Omega\right)^{\frac{1}{p}} \leq C_{p} .
$$

Taking $t_{0}=\frac{p-1}{p} \delta_{0}$ and using Hölder's inequality, we have

$$
\int_{M} e^{-t \phi},\left|s_{E}\right|_{h_{E}}^{-2 \frac{1-t}{\lambda}}\left|s_{D}\right|_{h_{D}}^{-2(1-\beta)} \Omega \leq A^{\frac{p-1}{p}} C_{p}
$$

It follows that for $t \in\left(0, t_{0}\right)$,

$$
F_{t} \geq J-C
$$

From this, we know that $A u t^{0}(M, E+D)=1$. Now put

$$
I=\{t \in[0,1] \mid(37) \text { is solvable }\} .
$$

By Lemma 5.3 and Theorem A in [GP], we know that $\left[0, t_{0}\right) \subset I$. Since there is no non-trivial holomorphic vector field tangential to $E$ and $D, I$ is open. We are going to show that $I$ is closed using log K-polystability.

Assuming that $\left(M, \omega_{t_{i}}, \frac{1-t_{i}}{\lambda} E+(1-\beta) D\right)$ converges to a metric space $(X, d)$ in the Gromov-Huasdroff topology as $t_{i} \in I \rightarrow T$. From Corollary 4.19, for some large integer $\ell$, we can embed $M$ into $\mathbb{C P}^{n}$ using orthonormal bases of $H^{0}\left(M, L^{\ell}\right)$ with respect to $\omega_{t_{i}}$, and we can also show that $(X, d)$ is homeomorphic to the Chow limit 
$\left(M_{\infty}, \omega_{\infty}, \frac{1-T}{\lambda} E_{\infty}+(1-\beta) D_{\infty}\right)$ which is a klt $\log$ Fano variety with weak conic Kähler-Einstein metric: .

Lemma 5.4. $A u t^{0}\left(M_{\infty}, \frac{1-T}{\lambda} E_{\infty}+(1-\beta) D_{\infty}\right)$ is reductive.

If $T=1, A u t^{0}\left(M_{\infty}, \frac{1-T}{\lambda} E_{\infty}+(1-\beta) D_{\infty}\right)$ is understood as $A u t^{0}\left(M_{\infty},(1-\beta) D_{\infty}\right)$.

Proof. We will use the method for proving Lemma 6.9 in [Ti15]. We will prove that any holomorphic field in the Lie algebra $\eta_{\infty}$ of $A u t^{0}\left(M_{\infty}, \frac{1-T}{\lambda} E_{\infty}+(1-\beta) D_{\infty}\right)$ is a complexification of a Killing field on $\left(M_{\infty}, \omega_{\infty}\right)$.

We only consider $T<1$, since the proof for $T=1$ is identical. Now $M_{\infty}$ is embedded in $\mathbb{C P}^{N}$ by $H^{0}\left(M_{\infty}, L^{\ell}\right)$. If $X \in \eta_{\infty}$ is a holomorphic vector field in $\mathbb{C P}^{N}$, then there is a bounded function $\theta_{\infty}$ satisfying

$$
i_{X} \omega_{\infty}=\sqrt{-1} \bar{\partial} \theta_{\infty}, \Delta \theta_{\infty}=-T \theta_{\infty}, \text { in } M_{\infty} \backslash \mathcal{S} .
$$

This is exactly the same as Lemma 6.9 in [Ti15]. Putting $\theta_{\infty}=u+\sqrt{-1} v$, we are going to show that $\nabla u$ corresponds to a Killing vector field. At first, we show that $u$ is the limit of eigenfunctions $u_{j}$ on $\left(M, \omega_{j}\right)$ such that $\Delta u_{j}=-\lambda_{j} u_{j}$ with $\lambda_{j} \rightarrow T$. Denote the set of such limit eigenfunctions by $\tilde{\Lambda}_{T}$ which is a subset of $\Lambda_{T}$ consisting of all bounded eigenfunctions with eigenvalue $T$. If $\tilde{\Lambda}_{T} \neq \Lambda_{T}$, we can find $u \in \Lambda_{T}$ such that

$$
\int_{M_{\infty}} u^{2} \omega_{\infty}^{n}=1, \quad \int_{M_{\infty}} u u_{a} \omega_{\infty}^{n}=0
$$

where $\left\{u_{a}\right\}_{1 \leq a \leq k}$ is an orthonormal basis of $\tilde{\Lambda}_{T}$. Because $\mathcal{S}$ is a subvariety which is contained in a divisor, as in Lemma 3.1, we have a cut-off function in $M_{\infty}$ satisfying

$$
\int_{M_{\infty}}\left|\nabla \gamma_{\epsilon}\right|^{2} \omega_{\infty}^{n} \leq \epsilon
$$

On the support $K_{\epsilon}$ of $\gamma_{\epsilon}$, by Lemma 4.3, we have $\phi_{j}: K_{\epsilon} \rightarrow\left(M, \omega_{j}\right)$ such that $\phi_{j}^{*} \omega_{j} \rightarrow \omega_{\infty}$ smoothly. So by taking a subsequence if necessary, we can take $\epsilon_{j} \rightarrow 0$ such that $u_{j}=\left(\gamma_{\epsilon_{j}} u\right) \circ \phi_{j}^{-1}$ satisfy

$$
\lim _{j \rightarrow \infty} \int_{M}\left|\nabla u_{j}\right|^{2} \omega_{j}^{n}=T, \lim _{j \rightarrow \infty} \int_{M} u_{j}^{2} \omega_{j}^{n}=1 .
$$

For each $a$, there are eigenfunctions $u_{a, j}$ of $\left(M, \omega_{j}\right)$ which converge to $u_{a}$. So if $j$ large enough, $u_{j}, u_{1, j}, \ldots, u_{k, j}$ is a $k+1$ dimensional subspace. We can find an eigenfunction $u_{0, j}$ orthogonal to $u_{a, j}(1 \leq a \leq k)$ with eigenvalue not bigger than $T+v_{j}$ with $v_{j} \rightarrow 0$. By Lemma 3.4, we know that the eigenvalue is not less than $t_{j}$. So $u_{0, j}$ will converge to an element in $\tilde{\Lambda}_{T}$. It leads to a contradiction. Now we know that $u$ is the limit of eigenfunctions $u_{j}$. By (16), we have

$$
\int_{M}\left|\bar{\nabla} \bar{\partial} u_{j}\right|^{2} \omega_{j}^{n} \leq\left(\lambda_{j}-t_{j}\right) \int_{M}\left|\nabla u_{j}\right|^{2} \omega_{j}^{n} \rightarrow 0
$$

It follows that $\bar{\nabla} \bar{\partial} u=0$ which means that $Y=\nabla u$ is a Killing vector field. As showed in Lemma 6.9 in [Ti15], $Y$ can be extended to a holomorphic vector field on $\mathbb{C P}^{n}$. So we have proved that $\eta_{\infty}$ is the complexification of the Killing vector fields. 
There is another approach to establishing the reductivity by using Theorem 5.1 in [BBEGZ] 2

By Luna's slice lemma (cf. [D] $)$, there is a test-configuration of $(M,(1-\beta) D)$ with $\left(M_{\infty},(1-\beta) D_{\infty}\right)$ as the central fiber. On $\left(M_{\infty}, \omega_{\infty}\right)$, the following Futaki invariant vanishes:

$$
F u t_{M_{\infty},(1-\beta) D_{\infty}}(X)-(1-T) \int_{M} \theta_{X} \omega^{n}+\frac{1-T}{\lambda} \int_{E} \theta_{X} \omega^{n-1}=0 .
$$

We will adapt the argument in $[\overline{\mathrm{DT}}]$ to prove this. Using the embedding of $H^{0}\left(M, L^{\ell}\right)$, we know that

$$
\omega_{j}=\frac{1}{\ell} \omega_{F S}-\frac{1}{\ell} \sqrt{-1} \partial \bar{\partial} \log \rho_{\ell}\left(M, \omega_{j}\right) .
$$

By Proposition 4.18 and gradient estimate, we know that $\rho_{\ell}\left(M, \omega_{j}\right)$ is uniformly Lipschitz. Denoting the limit of $\rho_{\ell}\left(M, \omega_{j}\right)$ by $\rho_{\ell}\left(M_{\infty}, \omega_{\infty}\right)$, we can also choose a bounded hermitian metric $h_{\infty}$ on the limit $\mathbb{Q}$-line $L_{\infty}=\frac{1}{\ell} O(1)$ with $R\left(h_{\infty}\right)=\omega_{\infty}$ in the regular part. Then we have

$$
\rho_{\ell}\left(M_{\infty}, \omega_{\infty}\right)=\sum_{i=0}^{N}\left|s_{i}\right|_{h_{\infty}^{\otimes l}}^{2} \text { for an orthonormal basis }\left\{s_{i}\right\} .
$$

For simplicity, denoting

$$
\bar{\omega}_{0}=\frac{1}{\ell} \omega_{F S}, \quad \varphi_{\infty}=-\frac{1}{\ell} \log \rho_{\ell}\left(M_{\infty}, \omega_{\infty}\right),
$$

we define

$$
\omega_{s}=\bar{\omega}_{0}+s \sqrt{-1} \partial \bar{\partial} \varphi_{\infty}=s \omega_{\infty}+(1-s) \bar{\omega}_{0} .
$$

The $\log$ Ricci potential $f_{0}$ of $\bar{\omega}_{0}$ is given by

$$
\sqrt{-1} \partial \bar{\partial} f_{0}=\operatorname{Ric}\left(\bar{\omega}_{0}\right)-T \bar{\omega}_{0}-\frac{1-T}{\lambda} E_{\infty}-(1-\beta) D_{\infty}
$$

We know that

$$
F u t_{M_{\infty},(1-\beta) D_{\infty}}(X)-(1-T) \int_{M} \theta_{X} \omega^{n}+\frac{1-T}{\lambda} \int_{E} \theta_{X} \omega^{n-1}=\int_{M_{\infty}} X\left(f_{0}\right) \omega_{0}^{n} .
$$

Put

$$
f_{s}=-\log \left(\frac{\omega_{s}^{n}}{\bar{\omega}_{0}^{n}}\right)-T s \varphi_{\infty}+f_{0}
$$

which is the $\log$ Ricci potential of $\omega_{s}$. Denoting by $v$ the potential of $X$ with respect to $\bar{\omega}_{0}$ :

$$
i_{X} \bar{\omega}_{0}=\sqrt{-1} \bar{\partial} v
$$

then we have

$$
i_{X} \omega_{s}=\sqrt{-1} \bar{\partial} \theta_{s}, \text { for } \theta_{s}=(1-s) v+s \theta_{\infty}=v+s X\left(\varphi_{\infty}\right) .
$$

From Lemma 5.4, we know that $|X|_{\omega_{\infty}}=\left|\nabla \theta_{\infty}\right|$ is bounded. Since

$$
\omega_{0} \leq C \omega_{\infty}, \quad s \omega_{\infty} \leq \omega_{s} \leq C \omega_{\infty},
$$

\footnotetext{
${ }^{2}$ In its new version posted in 2016, an appendix was added to fill in necessary arguments for a complete proof.
} 
we know that $|X|_{\omega_{s}} \leq C$ and $\left|\Delta_{\infty} v\right| \leq C$. Consequently, we have $\left|\Delta_{\infty} \theta_{s}\right| \leq C$ and $\left|\Delta_{s} \theta_{s}\right| \leq \frac{C}{s}$. Now

$$
X\left(\log \left(\frac{\omega_{s}^{n}}{\bar{\omega}_{0}^{n}}\right)\right)=\operatorname{div}_{s}(X)-\operatorname{div}_{0}(X)=\Delta_{s} \theta_{s}-\Delta_{0} v
$$

is bounded, so we have

$$
\begin{aligned}
\int_{M_{\infty}} X\left(f_{s}\right) \omega_{s}^{n} & =\int_{M_{\infty}}\left(\Delta_{0} v-\Delta_{s} \theta_{s}\right) \omega_{s}^{n}-T s \int_{M_{\infty}} X\left(\varphi_{\infty}\right) \omega_{s}^{n}+\int_{M_{\infty}} X\left(f_{0}\right) \omega_{s}^{n} \\
& =\int_{M_{\infty}} \Delta_{0} v \omega_{s}^{n}-T s \int_{M_{\infty}} X\left(\varphi_{\infty}\right) \omega_{s}^{n}+\int_{M_{\infty}} X\left(f_{0}\right) \omega_{s}^{n} .
\end{aligned}
$$

Taking $s \rightarrow 0$, we have

$$
\lim _{s \rightarrow 0} \int_{M_{\infty}} X\left(f_{s}\right) \omega_{s}^{n}=\int_{M_{\infty}} X\left(f_{0}\right) \omega_{0}^{n} .
$$

We are going to show that the integral

$$
\int_{M_{\infty}} X\left(f_{s}\right) \omega_{s}^{n}
$$

is independent of $s$. From $\frac{d}{d s} f_{s}=-\Delta_{s} \varphi_{\infty}-T \varphi_{\infty}$, we have

$$
\begin{aligned}
\frac{d}{d s} \int_{M_{\infty}} X\left(f_{s}\right) \omega_{s}^{n} & =\int_{M_{\infty}}\left[X\left(-\Delta_{s} \varphi_{\infty}-T \varphi_{\infty}\right)+X\left(f_{s}\right) \Delta_{s} \varphi_{\infty}\right] \omega_{s}^{n} \\
& =\int_{M_{\infty}}\left[\left(\Delta_{s} \theta_{s}+X\left(f_{s}\right)\right) \Delta_{s} \varphi_{\infty}-T X\left(\varphi_{\infty}\right)\right] \omega_{s}^{n} \\
& =\int_{M_{\infty}}\left[\Delta_{s} \theta_{s}+\theta_{s}+T X\left(f_{s}\right)\right] \Delta_{s} \varphi_{\infty} \omega_{s}^{n} .
\end{aligned}
$$

The integration by parts is guaranteed by the following two lemmas.

Lemma 5.5. $\left|\nabla \Delta_{s} \varphi_{\infty}\right|_{\omega_{s}}$ is bounded.

Proof. Putting $h_{s}=h_{\infty} e^{(1-s) \varphi_{\infty}}$, we have

$$
-s \varphi_{\infty}=\frac{1}{\ell} \log \left(\sum_{i=0}^{N}\left|s_{i}\right|_{h_{s}}^{2}\right)
$$

we also have

$$
\sqrt{-1} \partial \bar{\partial} \log \left(\sum_{i=0}^{N}\left|s_{i}\right|_{h_{s}}^{2}\right)=\sqrt{-1} \frac{\sum_{i=0}^{N}\left\langle\nabla s_{i}, \nabla s_{i}\right\rangle}{\sum_{i=0}^{N}\left|s_{i}\right|_{h_{s}}^{2}}-\sqrt{-1} \frac{\sum_{i=0}^{N}\left\langle\nabla s_{i}, s_{i}\right\rangle \sum_{i=0}^{N}\left\langle s_{i}, \nabla s_{i}\right\rangle}{\left(\sum_{i=0}^{N}\left|s_{i}\right|_{h_{s}}^{2}\right)^{2}}-\omega_{s} .
$$

From these, we can deduce that $\left|\nabla \Delta_{s} \varphi_{\infty}\right|_{\omega_{s}}$ can be bounded by the gradient of $s_{i}$. The lemma is proved.

Lemma 5.6. If $|\nabla u|_{\omega_{s}},|\nabla v|_{\omega_{s}}$ and $\left|\Delta_{s} u\right|$ are all bounded, then

$$
\int_{M_{\infty}}\langle\nabla u, \nabla v\rangle \omega_{s}^{n}=-\int_{M_{\infty}} v \Delta u \omega_{s}^{n}
$$


Proof. Let $\gamma_{\epsilon}$ be the cut-off function on $M_{\infty}$ as in Lemma 5.4, We have

$0=\int_{M_{\infty}} \operatorname{div}\left(\gamma_{\epsilon} v \nabla u\right) \omega_{s}^{n}=\int_{M_{\infty}} \gamma_{\epsilon}\langle\nabla u, \nabla v\rangle \omega_{s}^{n}+\int_{M_{\infty}} v\left\langle\nabla \gamma_{\epsilon}, \nabla u\right\rangle \omega_{s}^{n}+\int_{M_{\infty}} \gamma_{\epsilon} v \Delta_{s} u \omega_{s}^{n}$.

Since

$$
\int_{M_{\infty}} v\left\langle\nabla \gamma_{\epsilon}, \nabla u\right\rangle \omega_{s}^{n} \leq C \int_{M_{\infty}}\left|\nabla \gamma_{\epsilon}\right|^{2} \omega_{s}^{n}
$$

the lemma is proved by taking $\epsilon \rightarrow 0$.

A direct computation shows that $\bar{\partial}\left(\Delta_{s} \theta_{s}+\theta_{s}+T X\left(f_{s}\right)\right)=0$, so $\Delta_{s} \theta_{s}+\theta_{s}+T X\left(f_{s}\right)$ is a bounded holomorphic function which must be constant. From (39), we know that

$$
\frac{d}{d s}\left(\int_{M_{\infty}} X\left(f_{s}\right) \omega_{s}^{n}\right)=0
$$

Since $\omega_{\infty}$ is a weakly conic Kähler Einstein metric, $f_{1}=0$ and we have

$$
F u t_{M_{\infty},(1-\beta) D_{\infty}}(X)-(1-T) \int_{M} \theta_{X} \omega^{n}+\frac{1-T}{\lambda} \int_{E} \theta_{X} \omega^{n-1}=0 .
$$

The vanishing of $\log$ Futaki invariant can be also obtained from the $\log K$-polystability of singular varieties admitting weakly conic Kähler Einstein metric (Theorem 4.8 in [Ber $]$ ).

Denote

$$
f(t)=F u t_{X_{\infty},(1-\beta) D_{\infty}}-(1-t) \int_{M} \theta_{X} \omega^{n}+\frac{1-t}{\lambda} \int_{E} \theta_{X} \omega^{n-1}
$$

then from Lemma 5.3, we know that $f(t)>0$ for $t$ small. Since $f(T)=0$, we must have $f(1) \leq 0$. Because $(M,(1-\beta) D)$ is log K-polystable, $f(1)$ must be equal to 0 and $X_{\infty}$ is bi-holomorphic to $M$. It follows that $T \in I$ which means that $I$ is closed. So $I=[0,1]$ and we get the existence of conic Kähler-Einstein metrics.

\section{REFERENCES}

[An] Anderson, M. T.: Ricci curvature bounds and Einstein metrics on compact manifolds. J. Amer. Math. Soc., 2 (1989), no. 3, 455C490.

[Ber] Berman, R.: K-polystability of $\mathbb{Q}$-Fano varieties admitting KählerEinstein metrics. Invent. Math., (203) 2016, Issue 3, 973C1025.

[BBEGZ] Berman, R.; Boucksom, S.; Eyssidieux, P.; Guedj V. and Zeriahi A.: Kähler-Einstein metrics and the Kähler-Ricci flow on log Fano varieties. arXiv:1111.7158

[BBGZ] Berman, R.; Boucksom, S.; Guedj, V. and Zeriahi, A.: A variantioanl approach to complex Monge-Ampère equations. Pub. Math. de l'IHÉS, 117(2013), Issue 1, 179-245.

[Bo] Berndtsson, B.: Brunn-Minkowski type inequality for Fano manifolds and the Bando-Mabuchi uniqueness theorem. Invent. Math., (200) 2015, Issue 1, 149C200.

[B1] Blocki Z.: On the regularity of the complex Monge-Ampére operator, Complex geometric analysis in Pohang (1997) (Providence, RI), Contemp. Math., vol. 222, Amer. Math. Soc., 1999, pp. 181C189.

[BEGZ] Boucksom, S., Eyssidieux, P., Guedj, V. and Zeriahi, A.: Monge-Ampère equations in big cohomology classes. Acta Math., 205 (2010), Issue 2, 199-262.

[CC1] Cheeger, J. and Colding, T. H.: On the structure of spaces with Ricci curvature bounded below. I. J. Diff. Geom., 46 (1997), no. 3, 406C480.

[CC2] Cheeger, J. and Colding, T.H.: On the structure of spaces with Ricci curvature bounded below. II. J. Diff. Geom., 54 (2000), no. 1, 13-35. 
[CCT] Cheeger, J.; Colding, T. H. and Tian, G.: On the singularities of spaces with bounded Ricci curvature. Geom. Funct. Anal., 12 (2002), no. 5, 873-914.

[CDS15] Chen, X.X.; Donaldson S. and Sun S.: Kähler-Einstein metrics on Fano manifolds III: Limits as cone angle approaches $2 \pi$ and completion of the main proof. J. Amer. Math. Soc., 28 (2013), 235-278.

[Co] Colding, T. H.: Ricci curvature and volume convergence. Ann. of Math., (2) 145 (1997), no. 3, 477-501.

[DR] Darvas, T. and Rubinstein Y.: Tian's properness conjectures and Finsler geometry of the space of Kahler metrics. J. Amer. Math. Soc., 30 (2017), 347-387.

[DT] Ding, W.Y. and Tian, G.: Kähler-Einstein metrics and the generalized Futaki invariant. Invent. Math., 110 (1992), no. 2, 315-335.

[D] Donaldson, S.: Stability, birational transformations and the Káhler-Einstein problem. Surveys in Differential Geometry, 17. International Press, Boston, 2012.

[DS] Donaldson, S. and Sun, S.: Gromov-Hausdorff limits of Kähler manifolds and algebraic geometry. Acta Math., 213 (2014), no. 1, 63-106.

[GP] Guenancia, H. and Păun, M.: Conic singularities metrics with prescribed Ricci curvature: the case of general cone angles along normal crossing divisors. J. Diff. Geom., 103 (2016), no.1, 15-57.

[Li11] Li, C.: Remarks on logarithmic K-stability. Commun. Contemp. Math., 17 (2014), no. 2, 1450020, 1-17.

[S] Shen, L.M.: Smooth approximation of conic Kähler metric with lower Ricci curvature bound. Paci. J. Math., 284 (2016), no. 2, 455C474.

[Ti97] Tian, G: Kähler-Einstein metrics with positive scalar curvature. Invent. Math., 130(1997), no. $1,1-37$.

[Ti15] Tian, G.: K-stability and Kähler-Einstein metrics. Comm. Pure Appl. Math., 68 (2015), no. 7, 1085-1156.

[TW16] Tian, G and Wang, B.: On the structure of almost Einstein manifolds. J. Amer. Math. Soc. 28 (2015), no. 4, 1169-1209. 